\title{
Prominence marking in Parkinsonian speech and its correlation with motor performance and cognitive abilities
}

Tabea Thies ${ }^{1,4}$, Doris Mücke1, Anja Lowit² ${ }^{2}$ Elke Kalbe ${ }^{3}$, Julia Steffen4, Michael T. Barbe ${ }^{4}$ 1 IfL - Phonetics, University of Cologne, Germany

${ }^{2}$ School of Psychological Sciences and Health, University of Strathclyde, Scotland

${ }^{3}$ Department of Medical Psychology | Neuropsychology and Gender Studies \& Center for Neuropsychological Diagnostics and Intervention (CeNDI), Medical Faculty and

University Hospital Cologne, Germany

${ }^{4}$ Department of Neurology, Medical Faculty and University Hospital of Cologne, Germany

IfL - Phonetics

Faculty of Arts and Humanities

University of Cologne

Herbert-Lewin-Str. 6

50931 Köln

Germany

School of Psychological Sciences and Health

University of Strathclyde

40 George Street

G1 1QE Glasgow

Scotland

Department of Medical Psychology | Neuropsychology and Gender Studies \& Center for Neuropsychological Diagnostics and Intervention (CeNDI)

Medical Faculty and University Hospital Cologne

University of Cologne

Kerpener Str. 62

50937 Köln

Germany

Department of Neurology

Medical Faculty and University Hospital Cologne

University of Cologne

Kerpener Str. 62

50937 Köln

Germany

E-mails authors:

Tabea Thies (corresponding author): tabea.thies@uni-koeln.de

Doris Mücke: doris.muecke@uni-koeln.de

Anja Lowit: a.lowit@strath.ac.uk

Elke Kalbe: elke.kalbe@uk-koeln.de

Julia Steffen: julia.steffen@uk-koeln.de

Michael Barbe: michael.barbe@uk-koeln.de 


\begin{abstract}
Objectives

Research suggests that people with Parkinson's disease (PwPD) do not only suffer from motor but also non-motor impairment. This interdisciplinary study investigated how prominence marking is influenced by problems on the motoric and cognitive level.
\end{abstract}

\title{
Materials and methods
}

We collected speech production data from 38 native German speakers: 19 PwPD (under medication) with a mild to moderate motor impairment, 13 males and 6 females (mean 66.2 years old, SD = 7.7), and 19 healthy age- and gender-matched control participants (mean 65.4 years old, SD =9.3). Target words were produced in an accented and unaccented condition within a speech production task. The data were analyzed for intensity, syllable duration, F0 and vowel production. Furthermore, we assessed motor impairment and cognitive functions, i.e. working memory, task-switching, attention control and speed of information processing.

\section{Results}

Both groups were able to mark prominence by increasing pitch, syllable duration and intensity and by adjusting their vowel production. Comparisons between PwPD and control participants revealed that the vowel space was smaller in PwPD even in mildly impaired speakers. Further, task-switching as an executive function, which was tested with the trail making test, was correlated with modulation of F0 and intensity in PwPD: the worse the task-switching performance, the stronger intensity and F0 were modulated (target overshoot). Moreover, motor impairment within the PwPD group was related to a decrease in the acoustic vowel space (target undershoot), which further resulted in a decrease in speech intelligibility and naturalness. This behaviour of target over- and undershoot indicates an inefficient way of prominence marking in PwPD with mildly affected speech.

\section{Conclusion}

PwPD with signs of mild dysarthria did not differ from the control speakers with respect to their strategies of prominence marking. However, only the PwPD overused F0 and intensity in prominent positions. Overmodulation of F0 and intensity was correlated with the patient's task-switching ability and reflected abnormalities in the regulatory mechanism for expressing prosodic prominence. This is the first study to report a link between cognitive skills and speech production at the phonetic level in PwPD.

\section{Keywords}

Parkinson's disease; prominence marking; acoustic parameters; executive functions; motor functions; dysarthria 


\section{Introduction}

Human speech production involves complex interactions between the motor system and the cognitive system. Whereas, the motor system produces speech on the phonetic surface, the cognitive system is fundamental to produce motoric speech output in a contextually and grammatically correct way.

Prosody, as a suprasegmental feature of speech production, sits at the level above the production of individual sounds. Prosody conveys complex contextual information on different linguistic levels (Cole, 2015; De Looze et al., 2017), such as highlighting important information within an utterance, or distinguishing between a statement and a question, by modulating phonetic components; for example, fundamental frequency (F0) or intensity. Continuous adjustment of more than one phonetic component (Gafos et al., 2019) is generally necessary to signal the appropriate prosodic structures to support the intended meaning. Consequently, a dysregulation of this mechanism due to e.g. problems in motor performance and/or cognitive abilities can lead to difficulties in prosodic marking, resulting in a potential decrease in the degree of naturalness and speech intelligibility in every-day communication (Mücke et al., 2017).

The present study is concerned with the investigation of prominence marking (highlighting important words in a sentence) as a complex communicative function in speech in healthy people and people with Parkinson's disease (PwPD). The study investigates how Parkinson's Disease (PD) affects the balance of the speech motor system referring to the interplay of the different motoric subsystems of speech production at an early stage of dysarthria. Moreover, phonetic aspects of prominence marking will be correlated with motor performance and cognitive abilities.

Parkinson's Disease is a neurodegenerative disorder of the nervous system. Due to a progressive loss of dopaminergic brain cells in the substantia nigra, people who suffer from PD have problems with motor and non-motor functions (Ziegler \& Vogel, 2010; Skodda, 2015; Kalbe et al., 2016).

Motor symptoms such as rigidity and bradykinesia appear within the first five years of PD after diagnosis (Maetzler et al., 2009). Some patients develop a resting tremor and in later stages of the disease postural instability (Hornykiewicz, 1998; Koeslin, 2011). Clinical subtypes can be defined dependent on the most dominant motor symptom in the patient (Kang et al., 2005). An akinetic-rigid type would imply bradykinesia and rigidity resulting in slower, smaller and stiffer movements. A tremor-dominant type is characterised through a predominant resting tremor whereas an equivalent type is affected in all the three motor features.

Non-motor symptoms are frequently reported early symptoms in PwPD. Almost 20 to $33 \%$ of the patients have already cognitive dysfunctions at the time of diagnosis (Lawson et al., 2017; Santangelo et al., 2015). Many patients develop PD dementia in the course of the disease with an incidence of dementia, which is increased up to six times (Emre et al., 2007), and a cumulative prevalence of dementia up to $80 \%$ in 20-year survivors (Hely et al., 2008). Cognitive symptoms may include problems in the executive functions such as set-shifting, planning, strategy use and working memory. These symptoms are ascribed to dopaminergic dysfunction in the 'cognitive' frontostriatal circuit (de la Fuente-Fernández, 2012) as well as dysfunctions in attention and processing speed, memory and visuocognitive function.

Moreover, motor performance seems to be linked to cognitive abilities in PwPD. A study conducted by Salazar et al. (2017) postulated that dual tasking, which involves cognition and motor ability, affects walking speed. In addition, a recent study from Walton et al. 
(2018) discovered that gait disorders in PD are associated with cognition and that nonmotor (cognitive) training can improve gait performance.

One area that sits at the boundary between motor and non-motor impairment concerns communication. Communication is not only made up of speech, the production of words and sentences, but is also made up of language, which relates to the planning and understanding of what is said. Speech and language production are closely related to cognition, requiring cognitive skills such as memory, attention, information processing and planning. Although these symptoms are not always easily noticed, it is generally recognized that PwPD experience problems with language production and comprehension (Altmann \& Troche, 2011; Kalbe et al., 2016). However, there are still uncertainties about the exact relationship between cognition and language impairment. In contrast, speech impairment, which is closely linked to motor deficits in PD, is immediately obvious to the listener. 70 to $90 \%$ of PwPD will develop dysarthric speech at some point, generally of the hypokinetic type (Darley et al., 1969; Ho et al., 1999b; Duffy, 2013).

Hypokinetic dysarthria affects all motoric subsystems of speech production - glottal, subglottal and supraglottal - involving respiration, phonation, resonance and articulation (Darley et al., 1975; Ziegler \& Vogel, 2010; Skodda, 2015).

PwPD tend to experience a reduction in breath support, caused by the hypokinesia of the respiratory musculature. A reduced breath support results in quiet speech (hypophonia), monoloudness (Countryman \& Ramig, 1993, Holmes et al., 2000, Ramig et al., 2001) as well as shorter phrase length and a higher number of often inappropriately placed pauses (Ziegler \& Vogel, 2010; Skodda, 2015). Furthermore, an overall slowingdown of speech rate is described within the speech production of PwPD. This global slowing-down effect related to bradykinesia is usually measured in terms of syllables per second in fast syllable repetition tasks. Prolonged syllables are described as an indicator of dysarthria (Ackermann et al., 1995).

At the phonatory level, effects on voice quality (dysphonia) can be observed. PwPD are often presenting with a harsh and breathy voice quality and an associated pathology of vocal fold bowing. In addition, the ability to control vocal fold tension is impaired, leading to monotonous speech (monopitch) (Darley et al., 1975; Ziegler \& Vogel, 2010; Koeslin, 2011). Problems with resonance are not a primary symptom of PD but a mild degree of hypernasality can be present in patients.

PwPD show not only smaller but also slower movements of articulators (Ackermann \& Ziegler, 1991; Ziegler \& Vogel, 2010; Tykalova et al., 2017). Reduced articulation results in an articulatory undershooting/ undermodulation of phonetic parameters (Darley et al., 1975), which causes imprecise production of consonants. These smaller amplitudes of movements are in line with studies showing a reduced and centralized vowel space (Sapir et al., 2003, 2010; Hsu et al., 2017; Rusz et al., 2013b; Whitfield \& Goberman, 2014). In contrast, an articulatory overshoot/ overmodulation of phonetic parameters would be characterized through a more distinct articulation in the vocal tract and an increase of contrasts in the acoustic and perceptual space.

The onset and progress of these symptoms differs across patients, but phonatory insufficiency, reduced F0 modulation and reduced movement amplitude of the articulators are often reported already at early stages, often in the absence of significant intelligibility impairment (Rusz et al., 2013a). It should also be noted that symptoms can be highly variable across patients, e.g. not all PwPD exhibit hypophonia despite this being one of the most frequent signs of PD. Also rate patterns can vary from being 
abnormally slow, falling within the normal range to being accelerated (Forrest et al., 1989; Wong et al., 2011; Duffy, 2013; Gómez-Vilda et al., 2017).

One area that has been investigated in detail across all types of dysarthria is prominence marking. Prominence marking is a strategy for highlighting specific information important for communication. In intonation languages, such as German, prominence is marked on the postlexical level to single out relevant information. Prosodic highlighting generally involves dynamic changes in the glottal, subglottal and supraglottal system, i.e. intonation (speech melody), intensity as well as of movements of the articulators to change consonant and vowel characteristics in the prominent words.

Concerning intonation (i.e. the glottal level), the most prominent word in an utterance receives a pitch accent while unaccented syllables do not. In German, rising pitch accents are particularly perceived as prominent (Baumann \& Winter, 2018; Braun \& Ladd, 2003). The modifications within a pitch accent type such as a tonal rise are gradient. The higher the peak of the rising contour in the F0 trace (tonal height) and the later the alignment of the peak relative to the production of the target word on the segmental string, the greater the perceived prominence of the word (Gussenhoven, 2004; Baumann \& Röhr, 2015).

Concerning the subglottal system, prominence is associated with an increase in subglottal pressure, which leads to higher intensity values in the accented syllable. However, there is debate on whether intensity is one of the primary prominence markers, with some studies reporting little contribution (e.g. Kochanski et al., 2005), and others identifying intensity as the perceptually most salient parameter (cf. Lowit et al., 2018).

Finally, at the supraglottal level, changes of articulatory parameters, such as duration, velocity and amplitude of the movements, as well as a modified place of articulation (Mücke \& Grice, 2014) can be observed, particularly regarding the vowel space (Baumann et al., 2007). In a prominent position, vowels are articulated as clearly and distinctly as possible (Heuven \& Sluijter, 1996), by increasing distances in the acoustic space. Increased distances or increased contrasts on the paradigmatic axis are enhancing phonological features within perceptual impression. For example, the front vowel /i/ can be produced with a more fronted tongue position, the back vowel /o/ with a more retracted tongue position and the low vowel /a/ with a lower tongue position, all together increasing the vowel space area defined for the speaker (Kent \& Kim, 2003). These modulations are also gradient, i.e. the stronger the prosodic prominence (accentuation), and, therefore, the more focused the constituent, the more distinct the articulation. Furthermore, a prolongation of the entire syllable duration in prominent positions can be expected to increase the contrast between accented (prominent) and unaccented (non-prominent) syllables (Baumann et al., 2006, 2007; Kügler, 2008; Mücke \& Grice, 2016; Braun \& Ladd, 2003).

Note, that there is an interplay between the motoric subsystems of speech production, i.e. the subglottal, glottal and supraglottal system. Changes in one subsystem can also influence the processing in another subsystem as the output is the result of a complex filter system (Stevens, 2000). For example, overall intensity, which is related to the subglottal pressure, can also be influenced by both the glottal activity and the movements of the oral articulators in terms of how much energy can radiate from the mouth. However, the modulation of prominence can be attributed to phonetic cues that are often primarily related to one of the subsystems (as done throughout this manuscript): the intonational cues measured in the F0 traces are related to the glottal 
system. The temporal and spatial changes in consonant and vowel production on the paradigmatic axis are mainly related to the supraglottal system. Changes of overall intensity during syllable production are mainly related to subglottal modifications. In non-prominent (unaccented) positions, the physical control system tends to minimize the amount of articulatory effort for the different subsystems (Mattingly, 1981), a lowcost strategy, which is related to hypo-speech (hypo-articulation). In contrast, hyperspeech (hyper-articulation) leads to an increase of articulatory effort to signal prominence (de Jong, 1995; Harrington et al., 2000; Cho, 2006; Baese-Berk \& Goldrick, 2009; Scarborough, 2013; Mücke \& Grice 2014; Nelson \& Wedel, 2017). This leads to the use of multiple cues in the phonetic domain to regulate prosodic marking in a phrase (de Jong, 1995; Heuven \& Sluijter, 1996; Harrington et al., 2000; Cho, 2006; Mücke \& Grice, 2014; Mücke et al., 2017) and requires fine-tuning of enhancing and reducing costs of the speech system by modulating prosodic cues in a given utterance (Lindblom, 1990).

Studies on prominence marking in PwPD are rare, even though it has been shown that prominence marking can be impaired in PwPD even in the early stages of the disease due to deficits in the different subsystems required for speech production. Penner et al. (2001) investigated the F0 modulation in contrastive sentence and question production of 3 female German PwPD and found that, in line with the control speakers, they produced rising pitch accents to signal prominence, but presented with a smaller amplitude and earlier pitch peaks. Concerning loudness, Cheang and Pell (2007) showed lower intensity values on prominent syllables in the production of 21 male and female native English-speaking PwPD when producing mini-dialogues. Tykalova et al. (2014) investigated duration, intensity and pitch range in the production of 20 male Czech PwPD while reading text passages aloud containing target words in accented and unaccented condition. The PwPD modulated F0, intensity and durational properties in prominent positions, but not to the same extent as the healthy control participants. A sentence production experiment with varying target word position from Gaviria (2015) also focused on the three standard phonetic parameters and in addition investigated the vowel production. The results for 10 English-speaking PwPD (male and female) again demonstrated that the speakers were able to use intensity and durational differences to signal prominence. While duration and intensity were used to the same degree for prominence marking in PwPD and control participants, PwPD differed from the control group. PwPD made no adjustments within their vowel space and F0-contour. The studies thus suggest that PwPD can experience problems at all levels of prominence marking, but that there is strong variation in which phonetic parameters are impaired and to what degree.

Cognition and Prosody: Cognitive processes are involved in speech and language production to (i) plan an utterance, (ii) choose the linguistic units and (iii) initiate the right movements of the respective articulators in the glottal and supraglottal system (Levelt, 1989; De Looze et al., 2017). The first two steps are part of language production and well researched, the third is a matter of speech production, i.e. the planned output is translated into articulatory movements. To date, there is insufficient literature on the impact of cognition on stage (iii), although there are clear indications of a link.

There are several reports showing that speech rate is generally reduced in the context of increased task demands due to linguistic or cognitive task complexity (Swets et al., 2013) or the presence of parallel tasks (dual-tasking, e.g. Dromey \& Benson, 2003). In addition, Swets et al. (2007) described an effect of working memory on speech planning in terms of chunking an utterance. A decrease in working memory was associated with 
smaller phrase length in speech. Research also suggests that working memory skills impact on the amount of speech units that can be stored and the time needed from planning the speech output to executing it (Ferreira \& Swets, 2002; Levelt \& Meyer, 2000).

Further evidence for a link between cognition and speech production is presented by the study of impaired speaker populations. Filipe et al. (2018) investigated the relationship between executive functions and prosodic abilities in children with high-functioning autism. Their results revealed no correlation between prosodic abilities in terms of receptive and expressive prosodic skills (one total score for all skills) and speed of information processing, but showed a link to divided attention, working memory and set-shifting. Moreover, Rodgers et al. (2013) identified a relationship between a decrease in speed of information processing and slower speech rates in read and spontaneous speech in patients with Multiple Sclerosis, and De Looze et al. (2017) report similar results for pauses and sentence durations in the same population. Similar effects have been described for PwPD by Lowit et al. (2006), who found significantly slower speech rates in speakers with dementia with and without PD compared to healthy controls and cognitively healthy speakers with PD.

In addition to impacting on segmental and pause durations, cognition is likely to play an important role in prominence marking as this requires close monitoring of the discourse, as the transfer of information is dependent on the previous communication process. Information, which is already given in a conversation, is known by the attendees or present in their memory structure (Clark \& Haviland, 1977) and will be produced in background (unaccented) condition without much attention on the constituents. In contrast, discourse new and/or focused information is highlighted and activated in the listeners mind (Chafe, 1994) by modulating the properties of intonation and articulation. The challenge during the speech discourse is thus to maintain the attention on givenness and to translate this into the different prosodic structures.

The current literature includes variable reports on the ability of PwPD to mark prominence effectively. Whilst some of this variability will no doubt be due to differences in severity level of the studied speaker populations, the high prevalence of cognitive problems in PwPD and the suggested links between cognition and speech production, particularly in complex tasks such as prominence marking, suggests that cognition could also play a role in determining how effective speakers are in highlighting important information in their speech output. The aim of this study was therefore to investigate in more detail (1) the nature of prominence production in a phonetic way by measuring phonetic continuous variables in speakers with PD, and (2) the relationship between prosodic prominence and cognitive abilities.

To capture the cognitive abilities of the participants, executive functions in terms of working memory, speed of information processing and task-switching as well as attention control were assessed. Moreover, the speech performance was investigated using a self-rating tool to determine the impact of PD on speech production, rating the intelligibility and analyzing phonetic parameters associated with prominence marking.

The study investigated dynamic changes within the coordination of the speech subsystems reflected in phonetic exponents in prosodic highlighting strategies in PwPD and compared them to a group of age- and gender-matched healthy control speakers. The primary prominence markers related to the three subsystems of speech production were analyzed, i.e. F0 modulation, intensity, syllable duration and vowel production. 
Within each speaker group, the phonetic parameters were correlated with motor skills and cognitive functions. We have the following hypotheses based on the literature review for PwPD and control participants:

1) General speech performance: We expect to find a general decrease in the ability to adjust the phonetic parameters F0, intensity and the size of the acoustic vowel space in PwPD compared to control participants. Furthermore, we expect to find prolonged syllables in PwPD.

2) Prosodic marking: We expect that PwPD can manipulate F0, durational properties and intensity in accented syllables to express local prominence but will do so to a lesser extent than the control participants. In addition, we expect to find an undermodulation of the acoustic vowel space size by PwPD.

3) Association with motor performance: Prosodic highlighting strategies require fine motor performance of the speech system. We expect to find a correlation between motor impairment and the ability to manipulate syllable duration, intensity, tonal height and vowel production.

4) Association with cognitive functions: Since prosodic highlighting strategies require cognitive abilities that contribute to speech planning and execution, we expect to find a correlation between the level of cognitive skills and the signalling of prominence using the phonetic parameters: syllable duration, intensity, tonal height and vowel production.

a. Task-switching: Ability to switch between prosodic structure (background - given information vs focus - new information) is dependent on executive functions (divided attention, set-shifting). We hypothesize that a lowering of this ability will be associated with greater difficulties in producing prosodic prominence across all three speech subsystems.

b. Working memory: Lower working memory evokes problems to define given/new information within a speech discourse. These problems should either lead to an undermodulation of prosodic prominence on the correct word or to prominence on the wrong word.

c. Speed of information processing: The speed of information processing influences the speech rate. We expect that a decrease in the processing of information at the cognitive level will be associated with slower speech rates (here measured by syllable duration).

5) Intelligibility: We assume that with the overall motoric decline, speech production is also affected. Therefore, as motor impairment increases during the course of PD, the muscles of articulators will be impaired more heavily in later stages of the disease, reflected by a reduction in speech intelligibility. 


\section{Methods}

\subsection{Ethics}

The study was approved by the local ethics committee of the University of Cologne (17272). Participants gave a written informed consent before participating in the study.

\subsection{Participants and assessments}

38 native speakers of German were included in the study. Data were collected from 19 people suffering from Parkinson's Disease (mean 66.2 years old, SD $=7.7$ ) and from 19 healthy age- and gender-matched control participants (mean 65.4 years old, SD =9.3). Each group consisted of 13 males and 6 females, aged between 50 and 80 years (cf. table 1). An independent sample t-test confirmed that the patient group did not differ significantly from the control group in age ( $\mathrm{t}(34.86)=-0.3, \mathrm{p}>.05)$.

Inclusion criteria included German as native language and participants aged between 50 and 80 years. All patients needed a clinical diagnosis of the idiopathic Parkinson syndrome. Exclusion criteria included presence of dementia (established with the use of the Parkinson Neuropsychometric Dementia Assessment, PANDA, values below 14 indicate symptoms of dementia, Kalbe et al., 2008) and depression (Geriatric Depression Scale, GDS, values below 9 indicate none or only minimal symptoms of depression, Sheikh \& Yesavage, 1986; Arbeitsgruppe Geriatrisches Assessment, 1997). Informal assessment by an experienced speech and language therapist ruled out the presence of other speech and language problems such as aphasia, apraxia of speech or developmental disorders. No restrictions were applied to disease duration or the severity of dysarthria (cf. table 1, table 3) to allow us to investigate the impact of disease severity on the observed speech patterns.

The experiment took place in the department of Neurology of the University Hospital Cologne, Germany. Patients were recruited from our outpatient clinic. After determining inclusion status, PwPD were assessed by an experienced neurologist with the UPDRS III (Unified Parkinson Disease Rating Scale, Fahn et al., 1987; Goetz et al., 2008) as well as Hoehn \& Yahr scale (Hoehn \& Yahr, 1998) to determine severity of the disorder in relation to motoric impairment. The higher the values, the higher the motor impairment. In addition, the clinical subtype was defined to characterize which motor symptom was the most dominant in each patient (Kang et al., 2005; cf. table 1). Furthermore, the levodopa equivalent daily dose was calculated to determine the amount of levodopa drugs (LEDD, Tomlinson et al., 2010).

The speech recordings were performed immediately following motor examination to ensure as little fluctuation as possible in performance between gross motor and speech assessment. All PwPD were tested in their regular medication-ON condition ${ }^{1}$. Acoustic speech recordings were made using a condenser headset microphone in a soundattenuated room to optimize signal quality. The mouth-to-microphone distance of $7 \mathrm{~cm}$

\footnotetext{
1 We chose to enrol patients only in medication-ON condition, as this was a pilot study that aimed at determining for the first time whether there is a relationship between cognitive abilities and speech production. We also wanted to integrate patients with long-term illness into the study, but most of them are not able to participate in the OFF state, as symptoms are too strong, and patients are too heavily burdened. But we are aware that the dopa can potentially cover effects.
} 
as well as the microphone input settings were kept constant within speakers and across recording sessions. The acoustic signal was recorded at $44.1 \mathrm{kHz} / 16 \mathrm{bit}$.

Self-rating of voice impairment was captured with the voice handicap index (VHI, Jacobsen et al., 1997), where lower values represent a smaller handicap. The VHI was chosen as additional assessment giving an orientation about patient's speech problems and comparability as this test is often presented in speech studies. Furthermore, recordings of read speech using the standard text "The North Wind and the Sun" produced by the patients were rated by two trained listeners (one speech and language therapist and one trained phonetician) in terms of intelligibility and listener effort on a 1 to 9 scale (Dobinson, 2007), where 1 represents the poorest score, e.g. unintelligible, and 9 the highest (normal intelligibility) score.

\begin{tabular}{|c|c|c|c|c|c|c|c|c|c|c|c|c|}
\hline Patient & Sex & Age & $\begin{array}{l}\text { Clinical } \\
\text { subtype }\end{array}$ & $\begin{array}{l}\text { Dur. of } \\
\text { disease } \\
\text { (years) }\end{array}$ & LEDD & PANDA & GDS & Control & Sex & Age & PANDA & GDS \\
\hline 1 & $\mathrm{~m}$ & 54 & $a / r$ & 1 & 210 & 17 & 3 & \multirow{20}{*}{$\begin{array}{c}1 \\
2 \\
3 \\
4 \\
5 \\
6 \\
7 \\
8 \\
9 \\
10 \\
11 \\
12 \\
13 \\
14 \\
15 \\
16 \\
17 \\
18 \\
19 \\
\text { MEAN } \\
\text { (ISD) } \\
\end{array}$} & $\mathrm{m}$ & 50 & 15 & 0 \\
\hline 2 & $\mathrm{~m}$ & 56 & $\mathrm{a} / \mathrm{r}$ & 3 & 575 & 18 & 0 & & $\mathrm{~m}$ & 53 & 22 & 0 \\
\hline 3 & $\mathrm{~m}$ & 57 & $a / r$ & 2 & 626 & 27 & 4 & & $\mathrm{~m}$ & 54 & 27 & 0 \\
\hline 4 & $\mathrm{f}$ & 57 & $a / r$ & 9 & 490 & 25 & 3 & & $\mathrm{f}$ & 55 & 29 & 2 \\
\hline 5 & $\mathrm{~m}$ & 59 & $\mathrm{a} / \mathrm{r}$ & 1 & 780 & 22 & 0 & & $\mathrm{~m}$ & 56 & 27 & 2 \\
\hline 6 & $\mathrm{~m}$ & 61 & $\mathrm{e}$ & 5 & 475 & 26 & 2 & & $\mathrm{~m}$ & 60 & 17 & 1 \\
\hline 7 & $\mathrm{~m}$ & 62 & $a / r$ & 10 & 1475 & 26 & 5 & & $\mathrm{~m}$ & 62 & 29 & 0 \\
\hline 8 & $\mathrm{~m}$ & 62 & $a / r$ & 9 & 2130 & 26 & 7 & & $\mathrm{~m}$ & 62 & 25 & 0.5 \\
\hline 9 & $\mathrm{~m}$ & 65 & $\mathrm{a} / \mathrm{r}$ & 5 & 562 & 22 & 0 & & $\mathrm{~m}$ & 63 & 29 & 4.5 \\
\hline 10 & $\mathrm{f}$ & 67 & $\mathrm{a} / \mathrm{r}$ & 1 & 315 & 18 & 2 & & $\mathrm{f}$ & 64 & 20 & 0 \\
\hline 11 & $\mathrm{f}$ & 67 & $\mathrm{a} / \mathrm{r}$ & 10 & 765 & 23 & - & & $\mathrm{f}$ & 67 & 30 & 0 \\
\hline 12 & $\mathrm{~m}$ & 71 & $\mathrm{e}$ & 7 & 554 & 21 & 2 & & $\mathrm{~m}$ & 68 & 28 & 0 \\
\hline 13 & $\mathrm{~m}$ & 71 & $a / r$ & 8 & 705 & 17 & 5 & & $\mathrm{~m}$ & 71 & 23 & 2 \\
\hline 14 & $\mathrm{~m}$ & 73 & $a / r$ & 5 & 260 & 26 & 5 & & $\mathrm{f}$ & 72 & 26 & 0 \\
\hline 15 & $\mathrm{f}$ & 73 & $\mathrm{a} / \mathrm{r}$ & 5 & 454 & 29 & 2 & & $\mathrm{f}$ & 75 & 29 & 3 \\
\hline 16 & $\mathrm{f}$ & 73 & $\mathrm{a} / \mathrm{r}$ & 3 & 300 & 25 & 6 & & $\mathrm{f}$ & 76 & 17 & 2 \\
\hline 17 & $\mathrm{f}$ & 74 & $a / r$ & 14 & 510 & 23 & 2.5 & & $\mathrm{~m}$ & 77 & 26 & 2 \\
\hline 18 & $\mathrm{~m}$ & 76 & $\mathrm{a} / \mathrm{r}$ & 5 & 157 & 15 & - & & $\mathrm{m}$ & 78 & 21 & 1 \\
\hline 19 & $\mathrm{~m}$ & 80 & $\mathrm{a} / \mathrm{r}$ & 9 & 812 & 17 & 1 & & $\mathrm{~m}$ & 79 & 29 & 2.5 \\
\hline $\begin{array}{l}M E A N \\
( \pm S D)\end{array}$ & - & $\begin{array}{c}66.2 \\
( \pm 7.7) \\
\end{array}$ & - & $\begin{array}{c}5.9 \\
( \pm 3.7) \\
\end{array}$ & $\begin{array}{c}640 \\
( \pm 465) \\
\end{array}$ & $\begin{array}{c}22.3 \\
( \pm 4.2) \\
\end{array}$ & $\begin{array}{c}2.9 \\
( \pm 2.1) \\
\end{array}$ & & - & $\begin{array}{c}65.4 \\
( \pm 9.3) \\
\end{array}$ & $\begin{array}{c}24.7 \\
( \pm 4.7) \\
\end{array}$ & $\begin{array}{c}1.2 \\
(1.3) \\
\end{array}$ \\
\hline
\end{tabular}

Table 1: Participant characteristics about sex, age, values of dementia test (PANDA) and depression score (GDS). In addition, information for the patient group about Parkinson-type $(a / r=$ akinetic-rigid, $e=$ equivalent), duration of disease and levodopa daily dose (LEDD). '-'indicates missing data.

Additionally, cognitive abilities were examined: set-shifting ability, processing speech, attention control and working memory. Within the executive domain, the difference score of subtest B and A (B-A) of the trail making test (TMT) were used to assess taskswitching (testing the set-shifting domain). Subtest A of the TMT was used as an indicator for speed of information processing, where higher scores indicate lower processing speed as the time taken to finish the task was longer (Muir et al. 2015; $\mathrm{T}$ scores according to Tombaugh, 2004). Note that this latter score might be influenced by motor impairment in PwPD, although the TMT is strongly recommended for testing cognitive impairment in PwPD (Goldman et al., 2015). Furthermore, attention control was assessed with the Brief Test of Attention (BTA, Schretlen et al., 1996), working memory was examined with the digit span as part of the Wechsler memory scales (Wechsler, 1997). 


\subsection{Speech materials and data acquisition}

In order to analyze the prosodic marking strategies of PwPD and healthy control speakers, we investigated the production of target words in divergent prominence conditions: accented and unaccented. The speech task consisted of a question-answer scenario presented on a computer screen. Within the material the prominence status (accented and unaccented) of nine target words was manipulated by means of contextualizing questions (cf. table 2). These questions were presented visually and auditorily. The audio demonstrations were recorded previously to control the intonation of the question. The contrasting target word was emphasized to trigger the respective answer. While the question was orthographically presented on the screen, the target answer was indicated by an animation directly following the audio stimulus (cf. Example $1 \& 2$; underlined words in the questions represent highlighted constituents; capital letters show target words in contrastive focus condition receiving accentuation in appropriate answers).

\section{(1) Example 1: accented target noun}

Question: Hat die Fliege die grüne Nase berührt? ('Has the fly touched the green nose?') (Animation: Fly moves towards a green bee.)

Answer: Die Fliege hat die grüne BIENE berührt. (,The fly has touched the green BEE.')

(2) Example 2: unaccented target noun

Question: Hat die Fliege die grüne Biene berührt? ('Has the fly touched the green bee?') (Animation: Fly moves towards a brown bee.)

Answer: Die Fliege hat die BRAUNE Biene berührt. (,The fly has touched the BROWN bee.')

Target words (e.g. <Nase>;/'na:zə/.) were disyllabic (CV.CV structure), containing one of three long vowels /i:, a:, o:/ in the stressed syllable followed by an unstressed syllable. In German, the accented syllable of a target word in contrastive focus condition attracts accentuation, resulting in pitch accent placement, increased intensity and a greater vowel space. In total, we recorded 1368 tokens ( 9 target words x 38 speakers x 2 prominence conditions $\mathrm{x} 2$ adjectives).

\begin{tabular}{|c|c|c|c|c|c|c|c|}
\hline \multicolumn{2}{|c|}{ Adjective + } & \multicolumn{6}{|c|}{ Noun } \\
\hline & & \multicolumn{2}{|c|}{ /i:/ } & \multicolumn{2}{|c|}{ /a:/ } & \multicolumn{2}{|c|}{ /o:/ } \\
\hline \multirow{3}{*}{$\begin{array}{l}\text { grüne } \\
\text { (,green') } \\
\text { braune } \\
\text { (,brown') }\end{array}$} & \multirow{3}{*}{$\begin{array}{l}\text { gьу:nә } \\
\text { bьаữnə }\end{array}$} & $\begin{array}{l}\text { Biene } \\
\text { (,bee') }\end{array}$ & bi:nə & $\begin{array}{c}\text { Nase } \\
\text { (,nose') }\end{array}$ & na:zə & $\begin{array}{c}\text { Hose } \\
\left(, \text { trousers') }^{\prime}\right.\end{array}$ & ho:zə \\
\hline & & $\begin{array}{c}\text { Mine } \\
(\text {,mine })\end{array}$ & mi:nə & $\begin{array}{c}\text { Vase } \\
\text { (,vase') }\end{array}$ & va:zə & $\begin{array}{l}\text { Dose } \\
\text { (,can') }\end{array}$ & do:zə \\
\hline & & $\begin{array}{l}\text { Wiese } \\
\text { (grass') }\end{array}$ & vi:sə & $\begin{array}{l}\text { Wade } \\
\text { (,calf') }\end{array}$ & va:də & $\begin{array}{l}\text { Bohne } \\
\text { (,bean') }\end{array}$ & bo:nə \\
\hline
\end{tabular}

Table 2: Target words. Each adjective and noun given in German (bold), translated into English (in brackets) and written in phonetic notation (right).

\subsection{Speech data annotation and analysis}

The acoustic speech data were displayed and annotated by hand in Praat (Boersma \& Weenink, 2018; Version 6.0.37) by one trained phonetician (cf. figure 1). Every correctly produced target word (adjective and noun as well as the related segments of the noun) 
was annotated using the speech waveform and a wide-band spectrogram. Subsequently, its prominence status (accented vs unaccented) was determined perceptually by three trained phoneticians. Utterances with articulation errors and incorrectly produced prominence status (pitch accent on the wrong word) were excluded from the analysis of the phonetic parameters. Perceived errors in naturalness of production did not lead to exclusion. On average 3.4 productions out of 36 were excluded per control speaker and 3.5 productions per PwPD. An independent sample t-test verifies that the amount of incorrectly realized productions did not differ between the two groups $(\mathrm{t}(35.49)=-$ $0.18, \mathrm{p}>.05)$.

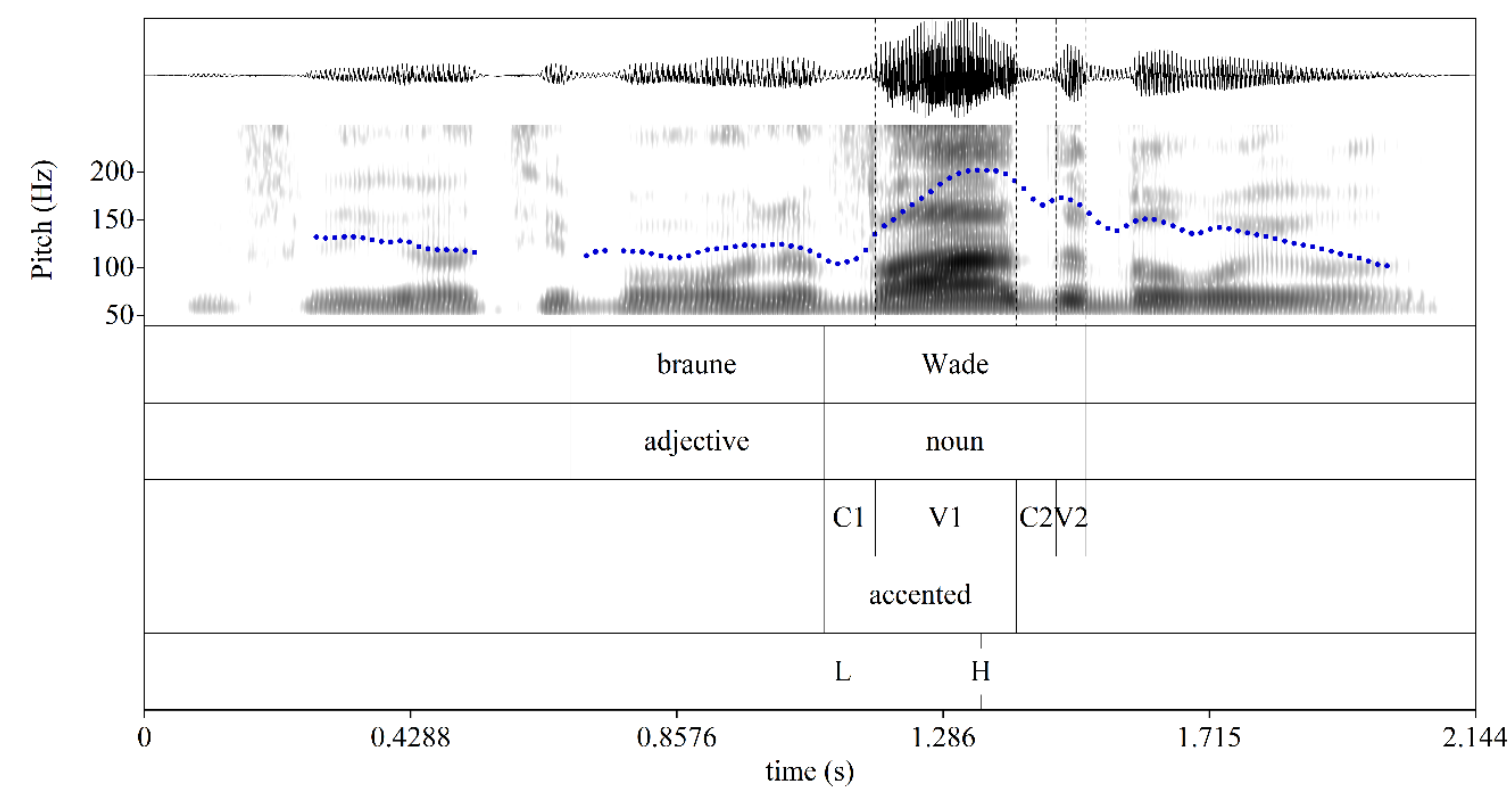

Figure 1: Annotation scheme in Praat. Acoustic signals at the top: speech waveform and a wide-band spectrogram and annotation levels at the bottom: words, part of speech, segments, accent condition of syllable and tones.

Data for intensity, fundamental frequency (F0), syllable duration and size of the vowel space were extracted, thus investigating the subglottal, glottal and supraglottal system. Acoustic vowel space was determined based on the vowel formant values for F1 and F2 in the accented and unaccented syllable and expressed as the Vowel Articulation Index (VAI, Roy et al., 2009; Sapir et al., 2010). This measure reflects vowel contrast and vowel centralisation and has been shown to be more sensitive to data with high variability and dysarthric vowel production compared to other common measures such as the acoustic vowel space area (VSA, Kent \& Kim, 2003; Vorperian \& Kent, 2007) (Sapir et al., 2011; Skodda et al., 2012). The procedure was as follows:

(1) Vowel formants (Hz): The mean vowel formants (F1, F2) taken from 5 measurement points within the vocalic segment V1 (/a, i, o/) of the target noun in accented and unaccented syllables were calculated. The mid-frequencies of the formants F1 and F2 are associated with the position of the respective vowel in the vowel space; F1 correlates with the opening of the vowel tract and F2 with the place of articulation.

(2) Vowel Articulation Index: The VAI was calculated using the following formula based on the formant values of F1 and F2 (Sapir et al., 2011). A decrease of the 
numerator and an increase of the denominator results in a reduced VAI and reflects a reduction in vowel space or greater centralisation (hypo-articulation of the supraglottal system). A higher VAI, on the other hand, shows an enhancement of the vowel space as expected during the marking of prominence (hyperarticulation).

$$
V A I=\frac{(F 2 / i /+F 1 / a /)}{(F 1 / i /+F 1 / o /+F 2 / o /+F 2 / a /)}
$$

Formant tracking errors of the LPC were fixed by adjusting the relevant present parameters such as number of total formants and maximum formant in $\mathrm{Hz}$ for extraction. The maximum value in $\mathrm{Hz}$ for female voices were $5500 \mathrm{~Hz}$ ( $5000 \mathrm{~Hz}$ for deep voices) and for male voices the value was $5000 \mathrm{~Hz}$ (4500 Hz for deep voices). In total 13 productions were excluded as calculation errors appeared even after settings were changed. Eight calculation errors were detected within the word 'Mine' across different speakers, although there was no difference between PwPD and healthy, controls.

Further measures included:

(3) Intensity $(\mathrm{dB})$ : The mean intensity (perceived loudness) of the vocalic segment V1 of a target noun in accented and unaccented syllables was determined. Sound pressure values were obtained by using the intensity algorithm in Praat that calculates dB SPL values relative to Praat's internal Pascal measurement (Boersma \& Weenink, 2014). Increased intensity values are associated with an increase in prominence marking (hyperarticulation of the subglottal system).

(4) Tonal height (st): The tonal height (or tonal range) is a spatial measure of F0 modulation. Therefore, we manually labelled the low starting point (L) and the high target point $(\mathrm{H})$ in the F0-contour of the pitch accent. In doing so, we identified turning points in the F0 trace for the respective rising pitch movements occurring in the vicinity of the target nouns. The corresponding frequency values for the rise contour were extracted in Hertz and converted to semitones to control for gender- and age-related differences in pitch. An increase in tonal height is associated with a higher degree of prominence in German intonation, reflecting hyperarticulation within the glottal system (Baumann \& Winter, 2018).

(5) Tonal alignment: Tonal alignment is a temporal measure to assess the coordination of the F0-contour relative to the boundaries of the accented target words. Therefore, we used the F0 landmarks for the low starting point (L) and the high target point $(\mathrm{H})$ for the nuclear rising pitch contours occurring in the vicinity of the target nouns. We computed latencies of the tonal events, $\mathrm{L}$ and $\mathrm{H}$, relative to segmental boundaries. The beginning $\mathrm{L}$ was measured as a proportion of the onset of the accented consonant $\mathrm{C} 1$ : alignment relative to start of $\mathrm{C} 1$, divided by total segment duration. The $\mathrm{H}$ was measured as a proportion of the onset of the accented vowel V1 in relation to the segment duration. These landmarks were chosen as previous studies showed for open CV syllables that L was aligned with C1 and H with V1 (D'Imperio, 2000, Prieto \& Torreira, 2007). 
(6) Syllable duration (ms): The syllable duration of the first syllable (start of first consonant C1 until end of first vowel V1) of each target noun was measured in accented and unaccented condition. Longer syllables are associated with an increase in prominence.

\subsection{Statistical analysis}

Group differences were investigated by conducting independent sample t-tests. Additionally, we investigated associations between paired samples, measuring correlation coefficients and linear mixed effect models with the lme4 package (Bates et al., 2015) in R Studio (RStudio Team, 2016; Version 1.1.447). All data files, two audio examples and the R codes used for the analysis of the results are provided on GitHub: https://github.com/TThies/Thies et al prominence marking in parkinsonian speech. In order to analyze the relationship between each continuous dependent variable (VAI, intensity, tonal height, syllable duration) and the critical predictors (group, prominence condition and further UPDRS III, cognitive scores, and intelligibility rating), we divided the linear mixed effect model analysis into two parts, (i) a group-based analysis where we checked for differences between the groups (control vs patient) and an influence of prominence condition (accented vs unaccented) considering individual speaker behaviour within the random structure, and (ii) an individual-difference analysis - one for each group - to investigate variations within one group by checking further influencing factors such as motor performance (UPDRS III), cognition and intelligibility.

In the group-based model we included the parameter 'group' as fixed factor and random slopes for 'speaker' and 'word'. In those cases where the influence of prominence condition was also investigated, the parameter 'accent' was included as a fixed factor and a within variable for 'accent per speaker' was added. A summary of the results is given in table 7 .

Models that tested individual differences were constructed with prominence condition and the parameter (z-standardized) of which the influence needed to be checked, and the random slopes for speaker (cf. table 8). Furthermore, the variance inflation factor (VIF) was calculated for testing collinearity between predictor variables. All tests resulted in an VIF of about 1 , revealing no collinearity between our chosen fixed factors.

The models were validated by comparing the test model (with the critical predictor) to a reduced model (without the critical predictor) via likelihood-ratio tests. P-values are based on these comparisons. Since we tested four different acoustic dependent variables, we corrected for multiple testing using the Dunn-Šidák correction, which lowers our analysis alpha level to $\mathrm{p}=0.0127$.

For some parameters (e.g. UPDRS III, intelligibility) we report relationships in terms of correlation coefficients, which are interpreted with the scale by Hinkle et al. (2002). We checked for a normal distribution of the data with the Shapiro-Wilk's test and used respectively the 'Pearson' or the 'Kendall' method and a confidence interval of $95 \%$ (cf. table 9). 


\section{Results}

\subsection{General assessment}

\begin{tabular}{|c|c|c|c|c|}
\hline Patient & UPDRS III & $\begin{array}{c}\text { Hoehn \& } \\
\text { Yahr }\end{array}$ & VHI & $\begin{array}{c}\text { Intelligibility } \\
\text { (1-9 scale) }\end{array}$ \\
\hline 1 & 18 & 2 & 9 & 7.25 \\
\hline 2 & 17 & 2.5 & 0 & 8.50 \\
\hline 3 & 15 & 1 & 5 & 9.00 \\
\hline 4 & 7 & 1 & 16 & 8.50 \\
\hline 5 & 8 & 1 & 18 & 8.75 \\
\hline 6 & 28 & 2.5 & 11 & 8.25 \\
\hline 7 & 22 & 4 & 54 & 8.00 \\
\hline 8 & 32 & 3 & - & 7.75 \\
\hline 9 & 35 & 2.5 & 2 & 8.00 \\
\hline 10 & 3 & 1 & 0 & 9.00 \\
\hline 11 & 29 & 3 & 14 & 8.00 \\
\hline 12 & 25 & 2 & 17 & 8.50 \\
\hline 13 & 40 & 3 & 49 & 6.50 \\
\hline 14 & 16 & 2 & 40 & 8.75 \\
\hline 15 & 20 & 2 & 51 & 7.20 \\
\hline 16 & 48 & 3 & 13 & 8.00 \\
\hline 17 & 28 & 3 & 0 & 8.75 \\
\hline 18 & 31 & 2 & 22 & 7.75 \\
\hline 19 & 30 & 3 & 55 & 7.25 \\
\hline $\begin{array}{r}M E A N \\
( \pm S D)\end{array}$ & $23.8( \pm 11.5)$ & $2.3( \pm 0.9)$ & $20.9( \pm 19.8)$ & $8.1( \pm 0.7)$ \\
\hline
\end{tabular}

Table 3: Patient characteristics about motor impairment (UPDRS III, Hoehn \& Yahr) and speech impairment (VHI = Voice Handicap Index, intelligibility ratings).

Motor scores: The motor performance in medication-ON condition was evaluated with the UPDRS III and Hoehn \& Yahr scale. A mean UPDRS III value of $23.8( \pm 11.5)$, ranging from 3 - 48 (out of max. 108 points to reach), and a mean Hoehn \& Yahr value of 2.3 ( \pm 0.9 ), ranging from $1-4$ (cf. table 3), were determined. These values indicate that the patient cohort shows a mild to moderate motor impairment with bilateral involvement.

General speech evaluation: The mean VHI values of the PwPD cohort were 20.9 ( \pm 19.8$)$, ranging from 0 - 55 (out of max. 120 point to reach), which means according to the VHI norm ranges that the self-perceived speech impairment ranged from mild to moderate. The intelligibility results confirmed this observation with a mean rating score of $8.1( \pm$ 0.7 ), ranging from $6.5-9.0$ (cf. table 3 ). The mean rating score of rater 1 was $8.2( \pm 0.6)$ and the mean score of rater 2 was $7.8( \pm 0.9)$, i.e. both speech experts agreed that some of the PwPD showed signs of mild dysarthria. While 13 PwPD were fully understandable without demanding much effort from the listener, 6 PwPD were mostly understandable, but the listeners needed to listen more carefully. Even though the two speech experts had a different professional background (one was a trained phonetician and the other a speech and language therapist), the rater agreement was acceptable, with the inter-rater reliability measure revealing a Cohen's kappa coefficient of $\mathrm{k}=0.41$. Additionally, the intra-rater reliability measure (Intraclass correlation coefficient) was strong for both raters (rater 1: 0.70; rater 2: 0.79), indicating that the presented results would be reproducible. 


\begin{tabular}{|c|c|c|c|c|c|}
\hline \multirow{2}{*}{ Domain } & \multicolumn{2}{|c|}{ controls $(n=19)$} & \multicolumn{3}{|c|}{ patients $(n=19)$} \\
\hline & Mean & $(S D)$ & Mean & $(S D)$ & $p$ \\
\hline \multicolumn{6}{|l|}{ Executive functions } \\
\hline \multicolumn{6}{|l|}{ Working memory } \\
\hline Digit span forward & 10.16 & $(2.01)$ & 9.05 & $(2.22)$ & \\
\hline Digit span backward & 6.90 & $(2.23)$ & 6.05 & $(1.99)$ & \\
\hline Digit span total score & 11.95 & $(2.78)$ & 10.63 & $(2.73)$ & \\
\hline \multicolumn{6}{|l|}{ Processing speed } \\
\hline TMT A & 31.90 & $(14.36)$ & 50.21 & $(21.58)$ & $* *$ \\
\hline TMT A, $P R$ & 68.95 & $(23.78)$ & 34.21 & $(29.50)$ & $* * *$ \\
\hline TMT B & 71.58 & $(38.99)$ & 133.61 & $(79.58)$ & $* *$ \\
\hline TMT B, $P R$ & 65.26 & $(30.25)$ & 31.67 & $(26.40)$ & $* * *$ \\
\hline \multicolumn{6}{|l|}{ Set shifting } \\
\hline TMT B-A & 39.68 & $(28.00)$ & 84.83 & $(65.04)$ & $*$ \\
\hline \multicolumn{6}{|l|}{ Attention } \\
\hline BTA A & 9.21 & $(1.13)$ & 8.78 & $(1.48)$ & \\
\hline BTA B & 9.16 & $(1.34)$ & 8.39 & $(1.82)$ & \\
\hline BTA total score & 18.37 & $(2.27)$ & 17.17 & $(3.17)$ & \\
\hline
\end{tabular}

Table 4: Cognitive measures. All values are raw values unless indicated otherwise. TMT = trail making test; $P R=$ Percentile rank; BTA = Brief test of attention. $\left({ }^{*} p<.05 ;{ }^{* *} p<.01 ;{ }^{* *} p<.001\right)$.

Cognition scores: Results of the TMT subtest A as an indicator of processing speed showed that PwPD were significantly slower $(\mathrm{t}(34.45)=4.0, \mathrm{p}<0.001)$ than the control participants, as they needed longer to complete the test. Also, the difference score (TMT subtest B minus A) as a measure for task-switching showed that the performance of PwPD was significantly reduced compared to the healthy control participants as the ttest verified $(\mathrm{t}(22.81)=-2.7, \mathrm{p}<0.02)$. Performance in all other tests, i.e. digit span as a measure for working memory and BTA as an indicator of attention control did not show significant differences.

\subsection{Vowel production and syllable duration}

Table 5, figure 2 and figure 3 present the results of the analysis for the patient group and the control participants. The values for the first two formants, F1 and F2, for the vowels /a, i, o/ as well as the computation of the respective vowel articulation index (VAI) and the syllable durations are provided in table 5 , separately for each prosodic condition (accented vs unaccented syllable). 


\begin{tabular}{|c|c|c|c|c|c|c|c|c|c|}
\hline \multirow{3}{*}{ group } & $\begin{array}{c}\text { accent } \\
\text { condition }\end{array}$ & $\begin{array}{c}\text { F1 } \\
\text { /a/ } \\
\text { (Hz) }\end{array}$ & $\begin{array}{c}\text { F2 } \\
\text { /a/ } \\
\text { (Hz) }\end{array}$ & $\begin{array}{c}\text { F1 } \\
\text { /i/ } \\
\text { (Hz) }\end{array}$ & $\begin{array}{c}\text { F2 } \\
\text { /i/ } \\
\text { (Hz) }\end{array}$ & $\begin{array}{c}\text { F1 } \\
\text { /o/ } \\
\text { (Hz) }\end{array}$ & $\begin{array}{c}\text { F2 } \\
\text { /o/ } \\
\text { (Hz) }\end{array}$ & VAI & $\begin{array}{c}\text { syllable } \\
\text { duration } \\
\text { (ms) }\end{array}$ \\
\hline \multirow{4}{*}{ patients } & \multirow{2}{*}{ unaccented } & 625 & 1274 & 276 & 2226 & 308 & 804 & 1.074 & 234 \\
& & $(182)$ & $(178)$ & $(48)$ & $(274)$ & $(56)$ & $(161)$ & $(0.109)$ & $(59)$ \\
\cline { 2 - 9 } & \multirow{2}{*}{ accented } & 657 & 1297 & 269 & 2269 & 331 & 748 & 1.113 & 259 \\
& & $(172)$ & $(171)$ & $(54)$ & $(268)$ & $(57)$ & $(111)$ & $(0.096)$ & $(67)$ \\
\hline \multirow{4}{*}{ controls } & \multirow{2}{*}{ unaccented } & 667 & 1250 & 259 & 2301 & 324 & 720 & 1.177 & 227 \\
\cline { 2 - 9 } & \multirow{2}{*}{ accented } & $735)$ & $(124)$ & $(51)$ & $(231)$ & $(56)$ & $(116)$ & $(0.092)$ & $(42)$ \\
& & $(121)$ & $(121)$ & $(63)$ & 2354 & 342 & 677 & 1.217 & 265 \\
& & $(250)$ & $(64)$ & $(111)$ & $(0.076)$ & $(67)$ \\
\hline
\end{tabular}

Table 5: Measurements for syllable durations and the formants F1 and F2 and the related vowel articulation index (VAI). Values indicate the calculated means and standard deviation is given in parenthesis.

Vowel production: All participants marked prominence in the segmental domain by adjusting formant frequencies of vowels in accented and unaccented positions. When comparing vowels in unaccented and accented syllables, the vowel articulation index (VAI) was increased in both PwPD and control participants. The PwPD increased their vowel space by about $3.6 \%$ and the control participants by about $3.4 \%$ in the accented compared to the unaccented condition, with the mixed model revealing an effect of prosodic condition on the VAI $\left(\mathrm{X}^{2}(1)=13.324, \mathrm{p}<.001\right)$. However, there were also group effects $\left(\mathrm{X}^{2}(1)=11.796, \mathrm{p}<.001\right)$ on the VAI calculations, i.e. the VAI was smaller in the PwPD than in the control participants in both test conditions (unaccented: smaller by $9.59 \%$, accented: smaller by $9.34 \%$ ).

\begin{tabular}{|c|c|c|c|c|c|c|c|c|c|}
\hline \multicolumn{10}{|c|}{$\mathrm{F} 2(\mathrm{~Hz})$} \\
\hline 30002500 & 2000 & 1500 & 1000 & 600 & 30002500 & 2000 & 1500 & 1000 & 600 \\
\hline \multicolumn{5}{|c|}{ unaccented } & \multicolumn{5}{|c|}{ accented } \\
\hline
\end{tabular}
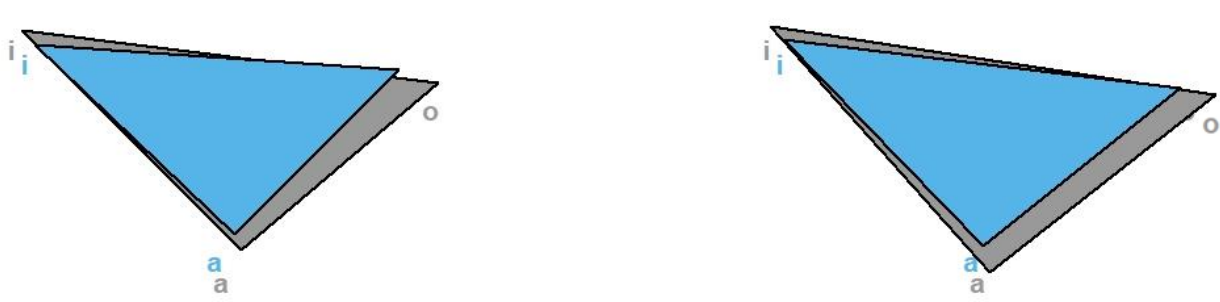

$\square$ Con $\square$ Pat

Figure 2: Triangular vowel space of the control participants (grey) and patients (blue).

The plotted formant values in figure 3 present that vowels in both accent conditions were produced with less variability in the control group than in the PwPD as the dispersion is smaller. In addition, both the back vowel /o/ and the low vowel /a/ were more centralized in the PwPD, as they partly overlap. 


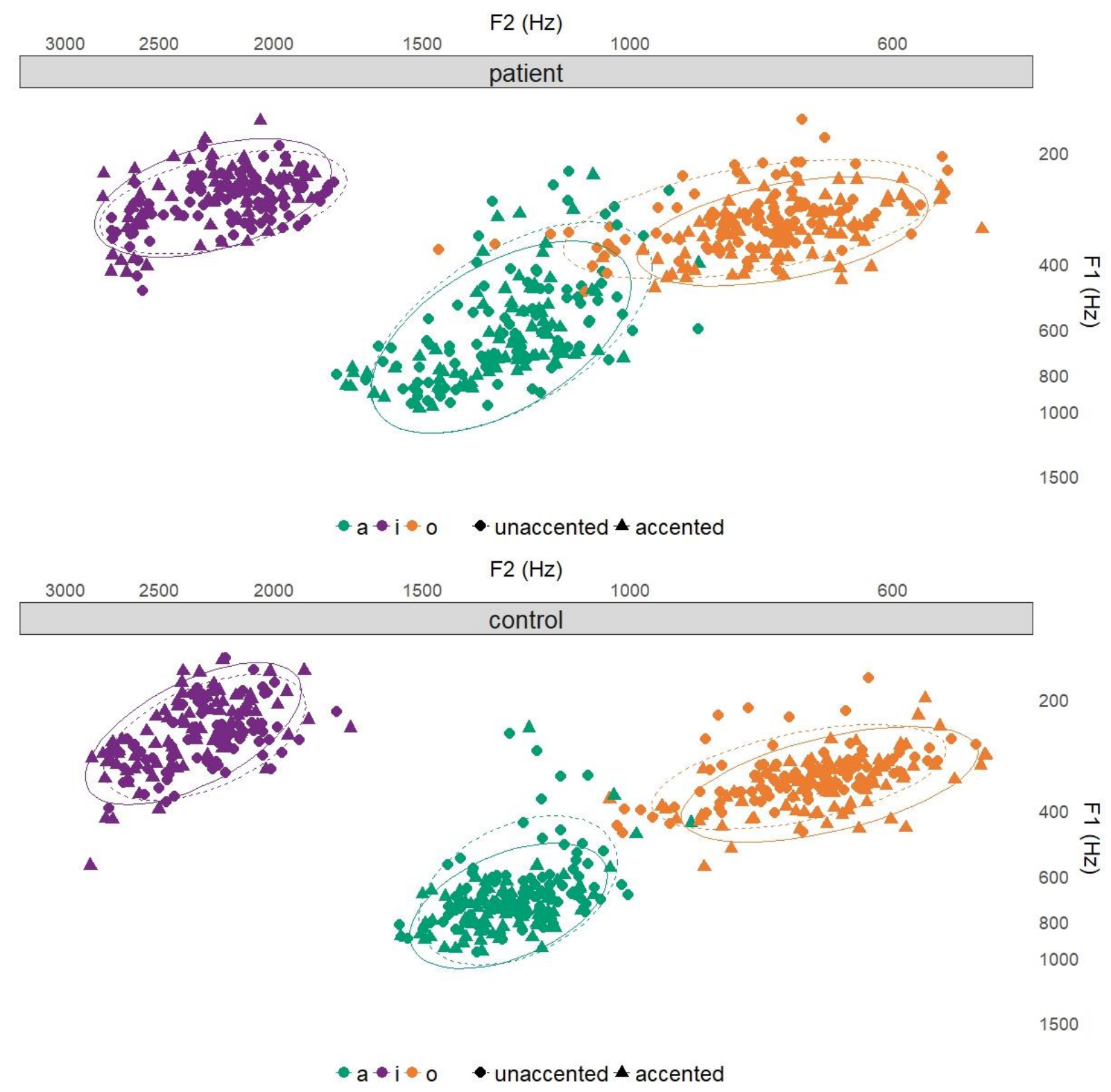

Figure 3: Formant values plotted as vowel charts, separately for the control participants (bottom) and the patient group (top). The solid lines and triangles represent the production in prominent position. Vowel / $\mathrm{i} /$ in purple, /o/ in orange and /a/in green.

Syllable duration: The analysis of syllable duration indicates that both participant groups produced longer syllables in the accented conditions (cf. table 5). A mixed model analysis reveals that there is an effect of prosodic condition $\left(X^{2}(1)=139.88, p<.001\right)$ but no group effect $\left(X^{2}(1)=0.0204, p>.05\right)$ suggesting that PwPD and control participants modulated syllable duration to the same extent. Note that the standard deviation values are comparable between the PwPD and the controls. This indicates that in our patient group no alternation between speeding up and slowing down on the global level of speech rate is present, as has been described in the literature for some studies with PwPD (Forrest et al., 1989; Wong et al., 2011; Duffy, 2013; Gómez-Vilda et al., 2017). 


\subsection{Intensity and F0}

Table 6 presents the group results for the intensity (subglottal) and F0 (glottal) measures. It shows values for the tonal height of the nuclear rise contour anchored in the vicinity of the accented syllable (in semitones). Furthermore, it shows intensity measures (in decibel) taken from the accented and unaccented target syllables to test whether speakers increased prominence by producing louder accented syllables.

\begin{tabular}{|c|c|c|c|}
\hline Group & $\begin{array}{c}\text { Tonal height } \\
\text { (st) }\end{array}$ & $\begin{array}{c}\text { accent } \\
\text { condition }\end{array}$ & $\begin{array}{c}\text { Intensity } \\
\text { (dB) }\end{array}$ \\
\hline \multirow{2}{*}{ patients } & \multirow{2}{*}{$7.0(3.0)$} & unaccented & $70(4.5)$ \\
\hline & & accented & $73(3.9)$ \\
\hline \multirow{2}{*}{ controls } & \multirow{2}{*}{$7.8(3.3)$} & unaccented & $68(4.6)$ \\
\hline & & accented & $72(5.2)$ \\
\hline
\end{tabular}

Table 6: Mean values (SD) of the tonal height and intensity. Decibel values were directly extracted from Praat.

Intensity: The intensity values show that both groups marked prominence by increasing the intensity level in the accented syllable, i.e. accented syllables were $3 \mathrm{~dB}$ louder in the patient group and $4 \mathrm{~dB}$ louder in the control participants. The statistical linear mixed model reveals an effect of accent status (accented vs unaccented syllables, $\mathrm{X}^{2}(1)=$ $520.26, \mathrm{p}<0.001$ ), increasing the sound pressure by about $3.2 \mathrm{~dB} \pm 0.01$ (standard errors). However, no systematic difference between the groups was found $\left(\mathrm{X}^{2}(1)=\right.$ $1.5732, \mathrm{p}>.05$ ).

Pitch: Figure 4 (left) presents violin plots with interquartile ranges and medians for the tonal height with means marked by the diamond. All speakers produced an intonational peak pattern in the vicinity of the accented target words (cf. figure 4 right). There was no group effect between PwPD and control participants on the tonal height of the nuclear rise as the mixed model reveals $\left(\mathrm{X}^{2}(1)=0.3345, \mathrm{p}>.05\right)$, i.e. both groups marked prominence by increasing tonal height to a similar extent (Con: $7.8 \mathrm{st}, \mathrm{SD}=3.3$; PwPD: $7.0 \mathrm{st}, \mathrm{SD}=3.0$ ). The rise in tonal height is also exemplified in the averaged F0contour co-occurring with the accented syllables, separately for the PwPD and the control participants (figure 4, right). The tonal rises are displayed on a normalized time axis, displaying the time interval between $50 \mathrm{~ms}$ before the start and $50 \mathrm{~ms}$ after the end of the accented syllable. The figure demonstrates that both cohorts exhibit a similar tonal range. However, when looking at the averaged contours of the intonational peak 
pattern in more detail, differences in the realization become apparent on the temporal axis.
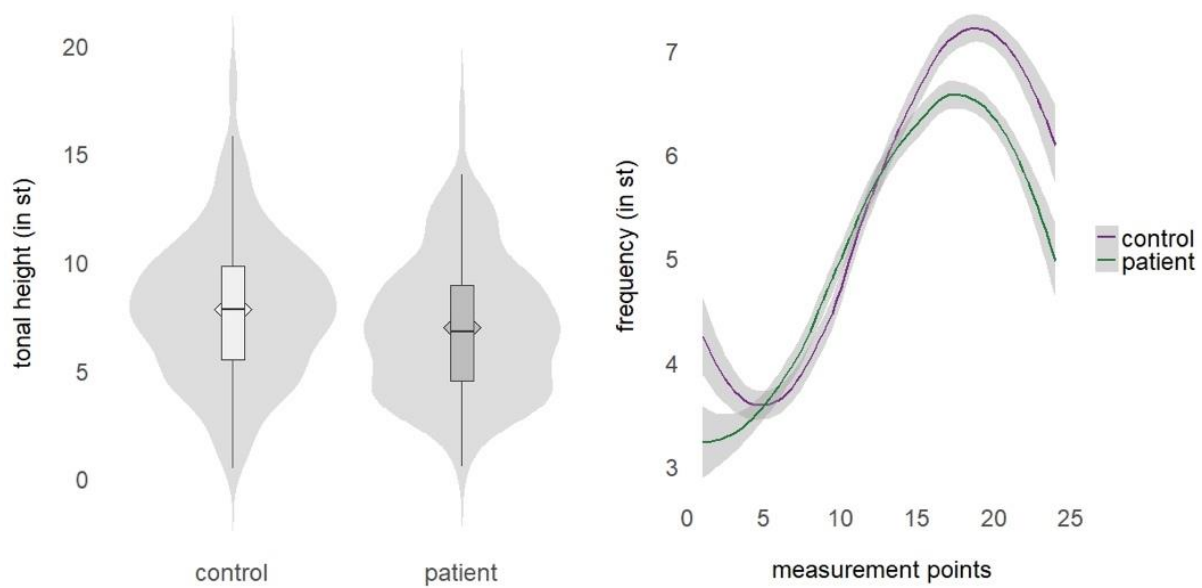

Figure 4: Violin plots with respective distributions for tonal height measures in accented condition for control participants and PwPDs (left) and the respective averaged F0-contours (right). The measurement points refer at 0 to a time point $50 \mathrm{~ms}$ before the accented syllable and at point 25 to $50 \mathrm{~ms}$ after the end of the syllable.

Consequently, we investigated the alignment patterns further by measuring the alignment of the low starting point (L) in relation to the duration of the consonant of the accented syllable. The results showed that both groups started the F0 movement in the middle of the consonant (Con: $53 \%$, SD = 38; PwPD: $50 \%$, SD = 95). The peak (H) was reached in the last quarter of the accented vowel but was timed slightly earlier in the PwPD (Con: $75 \%$, SD = 17; PwPD: $68 \%$, SD = 20). These results were confirmed by the statistical analysis, i.e. there were no group effects for the position of the low starting point $(\mathrm{L})$ relative to segmental boundaries of the accented target noun $(\mathrm{t}(236.18)=$ $0.4119, \mathrm{p}>0.05)$, but significant differences regarding the alignment of the F0 peak $(\mathrm{H})$ $(\mathrm{t}(373.45)=4.08, \mathrm{p}<0.001)$.

\begin{tabular}{|c|c|c|c|c|}
\hline & \multicolumn{2}{|c|}{ patients vs controls } & \multicolumn{2}{|c|}{ accented vs unaccented } \\
\hline $\begin{array}{c}\text { Dependent } \\
\text { variable }\end{array}$ & Fixed factors & p-value & Fixed factors & p-value \\
\hline VAI & \multirow{3}{*}{$\begin{array}{l}\text { (group) + accent } \\
\text { condition }\end{array}$} & 0.0005935 & \multirow{3}{*}{$\begin{array}{l}\text { group }+(\text { accent } \\
\text { condition) }\end{array}$} & 0.0002749 \\
\hline intensity & & 0.2097 & & $<2.2 \mathrm{e}-16$ \\
\hline syllable duration & & 0.8864 & & $<2.2 \mathrm{e}-16$ \\
\hline tonal height & (group) & 0.5573 & - & - \\
\hline
\end{tabular}

Table 7: Summary of group-based analysis. Differences between the speaker groups (patients vs controls) and the influence of prominence condition (accented v. unaccented) were tested. Random structure included individual speaker behaviour. The models were validated by comparing the test model (with the critical predictor) to a reduced model (without the critical predictor, here parameter in brackets) via likelihood-ratio tests. P-values are based on these comparisons.

\subsection{Correlation of variables}

To test our hypotheses $\mathrm{H} 3, \mathrm{H} 4$ and $\mathrm{H} 5$, we investigated the relationships between the phonetic prominence markers (VAI, intensity, tonal height and syllable duration) and potential influencing factors such as intelligibility rating, motor performance (UPDRS III) and cognitive abilities (TMT A, TMT B-A, BTA, digit span) for each participant group by performing linear mixed model analyses. The fixed factors were the same for each phonetic variable: Prominence condition (accented vs unaccented, except for tonal 
height) as well as the critical predictor of which the influence needed to be checked, were included (cf. table 8). Only significant results are reported to highlight the influencing factor for each group and parameter. A summary of all model comparisons is provided in table 8, which also presents the results for the control group. As can be seen, results were only significant for the PwPD group.

Vowel articulation index: The mixed model revealed that higher motor impairment (UPDRS III) was associated with a reduced VAI or smaller vowel space $\left(\mathrm{X}^{2}(1)=7.4147, \mathrm{p}\right.$ $<.001$ ) in the patient group (figure 5).

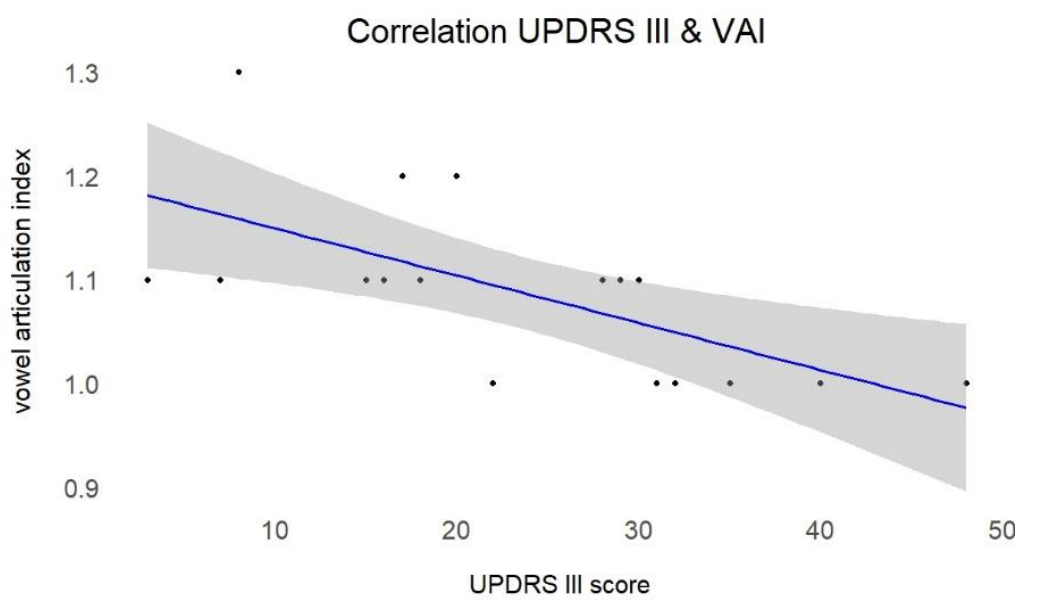

Figure 5: Negative correlation between motor impairment and vowel articulation index. Higher UPDRS III scores indicate a higher motor impairment. The shaded region depicts the confidence interval of $95 \%$ predicted by the model.

Intensity: Poor task-switching ability as an executive dysfunction (TMT B-A) in the PwPD was associated with an intensity overshoot $\left(\mathrm{X}^{2}(1)=8.1019, \mathrm{p}<.01\right)$, i.e. higher increases in sound pressure levels on accented syllables were associated with a decline in this cognitive function (figure 6).

\section{Correlation task-switching \& intensity}

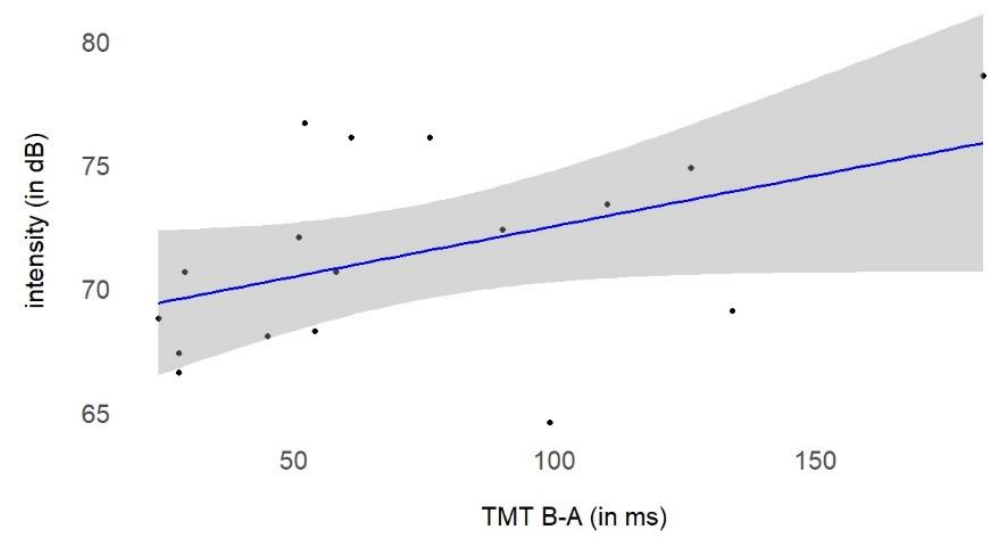

Figure 6: Positive correlation between task-switching ability and intensity values. Higher TMT B-A values indicate a slower performance (measured in $\mathrm{ms}$ ) and therefore a poorer task-switching ability. The shaded region depicts the confidence interval of $95 \%$ predicted by the model.

Tonal height: This parameter also correlated with task-switching, but not with any other predictors such as motor scores in the PwPD. Similarly, to the finding on intensity modulation a decline in task-switching ability (TMT B-A) was associated with an overshoot in tonal height $\left(\mathrm{X}^{2}(1)=7.2313, \mathrm{p}<.01\right.$, figure 7$)$. 


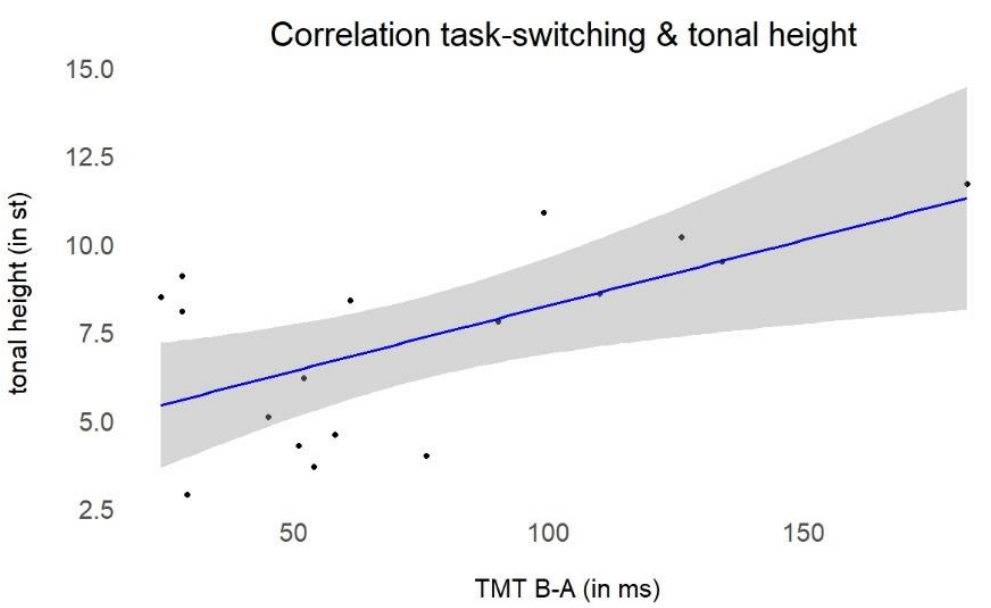

Figure 7: Positive correlation between task-switching ability and tonal height. Higher TMT B-A values indicate a slower performance (measured in $\mathrm{ms}$ ) and therefore a poorer task-switching ability. The shaded region depicts the confidence interval of $95 \%$ predicted by the model.

Syllable duration: The statistical analyses revealed no influencing factors for syllable duration for either of the participant groups.

\begin{tabular}{|c|c|c|c|c|c|c|c|c|}
\hline & \multicolumn{2}{|c|}{ VAI } & \multicolumn{2}{c|}{ Intensity } & \multicolumn{2}{c|}{ Tonal height } & \multicolumn{2}{c|}{$\begin{array}{c}\text { Syllable } \\
\text { duration }\end{array}$} \\
\hline $\begin{array}{c}\text { Critical } \\
\text { predictor }\end{array}$ & PwPD & Con & PwPD & Con & PwPD & Con & PwPD & Con \\
\hline TMT A & 0.04511 & 0.9552 & 0.1849 & 0.2786 & 0.7775 & 0.2647 & 0.204 & 0.4555 \\
\hline TMT B-A & 0.3465 & 0.3119 & 0.004422 & 0.7633 & 0.007164 & 0.4559 & 0.08805 & 0.6451 \\
\hline BTA & 0.0989 & 0.4656 & 0.1141 & 0.1196 & 0.01293 & 0.1829 & 0.2253 & 0.7485 \\
\hline digit span & 0.7006 & 0.5957 & 0.5377 & 0.3914 & 0.8477 & 0.549 & 0.5484 & 0.3797 \\
\hline UPDRS & 0.006469 & & 0.9033 & & 0.9012 & & 0.5312 & \\
\hline Intelligibility & 0.4054 & & 0.209 & & 0.282 & & 0.6344 & \\
\hline
\end{tabular}

Table 8: Summary of results from individual-difference-analysis. Given p-values are output of model comparisons, comparing model with critical predictor to a reduced model without critical predictor. Random structure included speaker. Alpha level: $p=0.0127$.

Further potential relationships between variables were investigated with a correlational analysis. The results are summarised in table 9. According to our hypothesis H5, we assessed whether speech intelligibility was correlated with motor impairment (UPDRS III). The Pearson correlation coefficient indicated that greater motor impairment was associated with worse intelligibility ratings (moderate negative correlation, $r=-0.56$ ). In addition, we correlated intelligibility with task-switching (TMT B-A), as this was implicated in the production of two phonetic prominence markers (intensity, F0). The relationship was determined using the 'Kendall' method, indicating that a decline in task-switching skills correlated with reduced speech intelligibility (low negative correlation, tau $=-0.30$ ). Interestingly, despite the observed relationships between intelligibility, cognitive and motor variables, neither the correlations between intelligibility and the phonetic prominence markers, nor the correlations between the UPDRS III and the TMT B-A scores were significant. Finally, the correlation analysis 
between the prominence markers also did not reveal any significant result for the PwPD (cf. table 9).

\begin{tabular}{|l|c|c|c|c|}
\hline \multicolumn{7}{|c|}{ patients } \\
\hline Correlation between: & method & coefficient & p-value & correlation \\
\hline Intelligibility $\sim$ intensity & kendall & -0.16 & $<0.001$, sign. & no \\
\hline Intelligibility $\sim$ tonal height & kendall & 0.16 & $<0.001$, sign. & no \\
\hline Intelligibility VAI & pearson & 0.24 & $>0.05$, n.s. & no \\
\hline Intelligibility syllable duration & kendall & -0.12 & $>0.05$, n.s. & no \\
\hline Intelligibility UPDRS & pearson & -0.56 & $<0.05$, sign. & moderate \\
\hline Intelligibility TMT B-A & kendall & -0.30 & $>0.05$, n.s. & low \\
\hline UPDRS $\sim$ TMT B-A & kendall & 0.19 & $>0.05$, n.s. & no \\
\hline VAI Intensity & pearson & -0.17 & $>0.05$, n.s. & no \\
\hline $\begin{array}{l}\text { VAI } \sim \text { Tonal height } \\
\text { (only accented condition) }\end{array}$ & pearson & -0.05 & $>0.05$, n.s. & no \\
\hline
\end{tabular}

Table 9: Summary of correlations of interest for the patient cohort. Pearson method was used for normally distributed data, Kendall for non-normally distributed data.

Significance of statistical models with and without outliers: The data for patient 13 were excluded from the analyses reported above as the score for the task-switching test (TMT B-A) reached considerably high values, considering this participant an outlier. If the data are included, the positively skewed distribution of the TMT values is shifted to the right (with patient 13: mean $=84.8, \mathrm{SD}=65$, without: mean $=73.4, \mathrm{SD}=44$ ). Given that most of our PwPD cohort had very mild impairment across speech, motor and cognitive components, it is worth considering this participant's performance in greater detail to model what happens in the context of more progressed decline of the task-switching ability.

\section{Correlation task-switching \& tonal height}

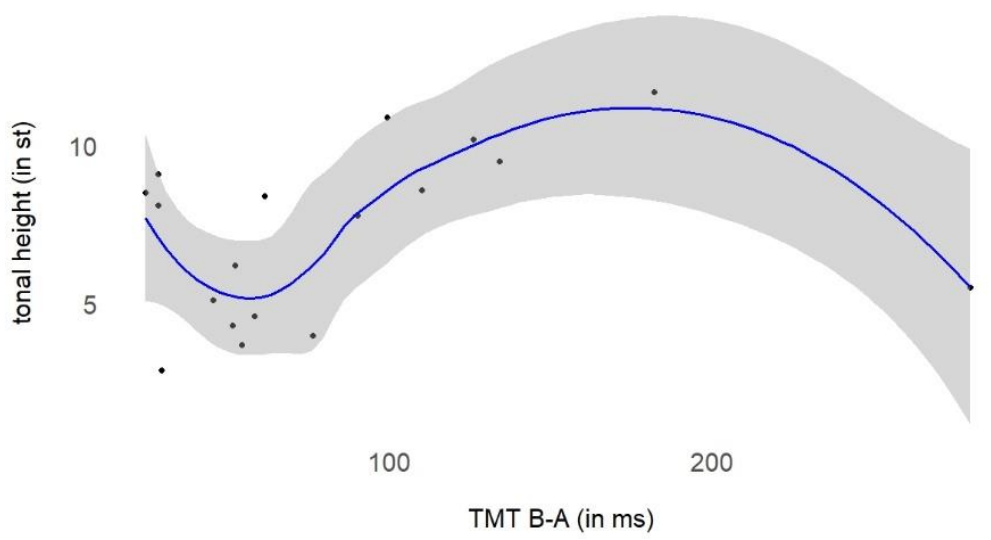

Figure 8: Correlation graph illustrating the relation between tonal height and executive functions in terms of task-switching ability (TMT B-A) with data from patient 13 included. Chosen method = loess. Higher TMT B-A values indicate a slower performance (measured in ms) and therefore a poorer task-switching ability. The shaded region depicts the confidence interval of $95 \%$ predicted by the model.

Therefore, we compared the statistical model with this patient as extra data point to the other original model. Again, we tested a linear regression behaviour of the parameters pitch height and intensity. The results show that the data without patient 13 is distributed in a more linear correlation because the AIC of the models is lower for the test without the extra data point in both cases, representing a better linear model fit (cf. figure 8 compared to figure 7). Based on this result one can hypothesize that the 
relationship between the executive function and the two phonetic parameters intensity and pitch changes if the cognitive dysfunction in terms of task-switching is getting worse. The mechanism of hyperarticulating pitch and intensity may change towards hypoarticulation of these parameters.

\subsection{Summary}

Our data revealed that control speakers and PwPD with mild dysarthria can produce prominence by modulating syllable duration, F0, intensity and vowel production. Whereas syllable duration, F0 and intensity are used to the same extent across the speaker groups, the vowel articulation index differed between the groups. PwPD had a smaller vowel space available in which they modulate articulatory parameters. The analysis of the cognitive status of the participants showed that the two groups differed significantly in speed of information processing (although the results of this test might have been influenced by motor impairment) as well as task-switching, but not in attention control and working memory. Analyzing the relationship between cognitive abilities and prominence production, we found that a decline in task-switching ability was associated with an increase/overmodulation of F0 and intensity in the PwPD. Additionally, overall motor performance correlated with the vowel articulation index of the PwPD, leading to a reduction (undermodulation) of the vowel space and a more centralized articulation, which was further associated with a lower degree of speech intelligibility.

\section{Discussion}

We investigated the ability and process of prominence marking in terms of modulation of F0, syllable duration, intensity and vowel space in a group of PwPD and healthy control participants and investigated whether motor performance and cognitive abilities as well as dysarthria severity had an impact on the ability to modulate these parameters.

General assessment of the participants: The UPDRS III in medication-ON condition indicates a mild to moderate motor impairment in the patient group (cf. table 3) and symptoms on both sides of the body according to the Hoehn \& Yahr scale. Although our participants were randomly selected, none of the patients had a tremor-dominant PD. Most of them were characterised with an akinetic-rigid PD and two patients with an equivalent PD type. Evaluating the speech ability, the results of the VHI and the intelligibility ratings suggest that most of the PwPD were generally considered as fully understandable and mildly dysarthric.

Assessment of the cognitive status revealed that the participant groups showed significantly different results testing executive functions in terms of task-switching (TMT B-A) and speed of information processing (TMT A), although, as indicated above, it cannot be ruled out that the latter score was influenced by motor impairment. The performance in attention control and working memory did not differ and was not affected in either group. As executive dysfunction belongs to the most frequent and vulnerable functions in PwPD (Kalbe et al., 2016), this can be regarded as a typical cognitive profile of PD. Working memory is also frequently affected, so that dysfunction in this domain could also be affected. However, it should be kept in mind that cognitive profiles in PwPD are highly heterogenous, which is reflected by the fact that all four types of MCI (amnestic single and multiple domain as well as non-amnestic single and multiple domain MCI) can occur, and the sensitivity to detect cognitive impairment differs (Kalbe et al., 2016). Notably, the trail making test is among the best ones rated in 
a recent paper examining MCI diagnosis according to the research criteria of the Movement Disorders Society (Goldman et al., 2015).

General speech performance: Based on the literature, a general decrease of glottal, subglottal and supraglottal speech production in PwPD can be expected, related to symptoms of hypokinetic dysarthric speech (Darley et al., 1975; Ziegler \& Vogel, 2010; Skodda, 2015). This was confirmed in our data for PwPD, who presented with smaller acoustic vocal space, less distinct vowel production and thus a greater centralisation of the vowels /i:, a:, o:/. In the PwPD, there was a considerable overlap of the vowel categories /a/ and /o/, especially in the unaccented condition. This was the case despite the relatively low level of gross motor as well as intelligibility impairment. Articulation of the vowels /a/ and /o/ involves movements of the tongue dorsum in the velar and pharyngeal region. Our results suggest that even mild speech motor impairment already has an influence on movements of the articulators in the back region of the oral tract, resulting in less distinct articulation, which mirrors results from Karlsson \& Hartelius (2019) who report the velar articulation place as the first domain to show changes in (mild) dysarthric patients with PD in a fast syllable repetition task.

On the other hand, we did not identify any differences in the overall use of loudness, durational properties and pitch between speaker groups. Therefore, we have to refute our hypothesis $\mathrm{H} 1$ for most of the phonetic parameters, as only vowel production was affected in our PwPD group.

Local prominence marking: It has been stated in the literature that the expected decrease in the ability of adjusting phonetic parameters in general due to deficits in the speech subsystems is also reflected in local prominence marking when comparing PwPD and control participants (Penner et al., 2001; Tykalova et al., 2014). Even though our data support the assumption that PwPD could highlight prominent elements in accented conditions by adjusting all subsystems in terms of glottal, subglottal and supraglottal control, the prosodic adjustments in the PwPD group differed from the control group.

The segmental analysis revealed that speakers in both groups increased the vowel space in prominent conditions to highlight the accented word. Indeed, the mean percentage range in resizing the vowel articulation index was slightly larger in the PwPD group than in the control group. However, it should be noted here that the vowels are already more centralized in unaccented condition in the productions of the PwPD group. The overall perceptual distances between the vowels were smaller in the PwPD group than in the controls, resulting in a decrease in paradigmatic contrasts between accented and unaccented words. Reduced paradigmatic contrasts fit with the results of the speech intelligibility testing, revealing a decrease in speech intelligibility due to motor impairment in Parkinsonian speech, likely due to a decrease in the ability of reaching vocalic targets in the oral tract. In contrast, durational properties such as the overall syllable duration of the stressed syllable in both accent conditions seemed to be unaffected.

For the suprasegmental analysis, a different picture arose. On the one hand, we found a similar range of intensity modulation in the PwPD and the control participants; in line with the results of Gaviria (2015), but contrary to Cheang and Pell (2007). Similarly, our groups did not differ in terms of pitch height in intonational peak patterns, again contradicting Cheang and Pell (2007), as well as Penner et al. (2001), Tykalova et al. (2014) and Gaviria (2015) who reported a reduced pitch height to express prominence. 
However, while the spatial domain did not differ, we found a difference in the timing of pitch accents relative to the accented target word in line with Penner et al. (2001). Compared to control participants, the PwPD group achieved the target of the rising F0contour earlier with respect to the highlighted target word. The analysis of Penner et al. (2001) indicates that this can lead to problems in the perception of intonational meaning, since medial and late peaks mark newness of information, and later peak placements in a nuclear rising accent in German are linked to perception of greater prominence of the target word (Gussenhoven, 2004; Baumann, 2014; Baumann \& Röhr, 2015). Production of early peaks could thus reduce the effectiveness of highlighting important information.

To explain this finding, one should consider that intonational alignment involves the coordination between different motor speech systems, requiring fine-tuning between the production of the F0-contour at the glottal level and the consonant-vowel production at the supraglottal level. We have seen that both systems are affected in different ways in our data: the PwPD showed an overmodulation of suprasegmental parameters associated with task-switching ability and an undermodulation of segmental parameters such as the vowel associated with motor impairment. Therefore, the coordination between the systems might have been affected leading to a change in alignment for PwPD.

Based on the analyses above, we must refute our hypothesis $\mathrm{H} 2$ with the exception of vowel production, and to some degree F0 modulation, although only in the temporal rather than the spatial domain.

In contrast to previous published studies on prominence marking in PwPD (Penner et al. 2001, Cheang and Pell, 2007, Tykalova et al. 2014), our study revealed that PwPD can produce prosodic prominence to the same extent as the control speakers. The modulation of F0, intensity and syllable duration did not differ across the groups. One explanation could be that most of our patients were only mildly dysarthric, performing with a less affected speech system than in other studies, and therefore were able to modulate the speech systems involved. Nevertheless, it might be that the controlled experimental set-up and the acoustic hints given in the auditorily presented questions led to the good performance. A study by Ho et al. (1999a) investigated that overall intensity comparing controls and PwPD were the same, but less in conversations for the PwPD. In conversations in which patients wanted to be heard on distance (reaching a goal) they were able to increase intensity. A good performance in paradigms with clues could explain why the ability to mark prominence was maintained in our patient cohort. However, another explanation could be that the experimental task might have been too easy for the patients to exhibit prosodic deficits. The low variation between the stimuli could have influenced our results as the participants got used to the schemata. But bearing in mind that dual tasking is difficult for PwPD, this might weaken this idea. Salazar et al. (2007) investigated that dual tasking had a significant effect on cognition (reduced set-shifting) and motor performance (walking) limiting the ability to perform complex tasks.

We assumed that working memory affects the memory structure and therefore the 'discourse knowledge' and the understanding of givenness. The data revealed neither group differences in relation to working memory nor according to the number of incorrectly accented words, most probably due to absence of cognitive problems in the first place (except set-shifting problems). Regarding the ability to analyze a speech discourse, we can conclude from the data that all participants still knew which 
constituents needed to be highlighted and how to realize this at the phonetic level. Therefore, the ability to contrast different prosodic structures was sustained in our cohort (cf. hypothesis H4a). Relating to our hypothesis, we have to deny any influence of working memory on the ability of prominence production (cf. hypothesis $\mathrm{H} 4 \mathrm{~b}$ ).

\section{Influence of executive functions and motor performance on phonetic parameters: We can} confirm, in line with our hypothesis $\mathrm{H} 3$, that articulation, expressed as the ability to expand the vowel space, is closely related to the level of motor disability in PD.

Previous studies also investigated that motor symptoms such as bradykinesia and rigidity are primarily responsible for reduced vowel production in PwPD (Skodda et al., 2012; Rusz et al., 2013a). Therefore, vowel production seems to be linked to these early motor symptoms, which are the first symptoms of the motor phase appearing within the development of PD (Kalia \& Lang, 2015). This fact explains why the vowel space is already reduced in PwPD with mild dysarthria in our data. No such relationships were apparent for intensity, syllable duration and F0 modulation. However, the interrelationship of the different subsystems was more complex when looking within the groups and correlating the respective speech production parameters with measures of motor performance (UPDRS III) and cognition (TMT B-A, task-switching). With respect to UPDRS III and TMT testing, we found that three speech subsystems were affected differently. On the one hand we determined that impaired task-switching was related to stronger modulation of intensity (subglottal) and F0 (glottal), confirming our hypothesis H4a. On the other hand, vowel production (supraglottal) was more associated with the motor impairment (cf. hypothesis H3). This led to an imbalance of the speech systems during prominence marking, resulting in an overshoot of pitch and intensity control, whilst the lingual productions were undershooted at the same time. This could suggest that PwPD made too much effort to modulate the prominence markers F0 and intensity, reducing their capacity to modulate oral articulation. Our results suggest that this was to some degree due to limitations in motor performance, and at the same time affected by cognitive function. One might argue that not only vowel production is dependent on motor function but also the control of F0 and intensity. However, in context of prominence marking, cognition seems to play an additional role.

We provide representative audio examples from a patient and a healthy control speaker on GitHub. Both speakers are able to mark prosodic prominence, but the patient's utterance sounds unnatural. The word "Wade" is very loud in contrast to other words within the utterance and the corresponding F0 modulation is rather strong. The prominence marking would have also worked successfully if the patient had modulated F0 and intensity to a lesser extent.

As reported above the modulation of syllable duration and therefore the speech rate seemed to be unaffected in both groups and did not show any correlation with the speed of information processing, although the processing speed of the patients was slower. Based on this result, we cannot confirm hypothesis H4c, i.e. the speed of information processing did not influence the speech rate.

Analysis of intensity and pitch parameters with outlier: As mentioned before, patient 13 was excluded from the main statistical analysis of the influence of executive functions due to his particularly high score in the task-switching test (TMT B-A $=280$ ). The patient's characteristics illustrate that he had PD for 8 years at the time of recording, including a moderate/severe motor and speech impairment (UPDRS III $=40, \mathrm{VHI}=49$ ) 
as well as a low intelligibility (value $=6.5$ ). An individual post-analysis of the phonetic parameters determined that patient 13 did not adjust the vowel articulation (VAI accented condition: 0.986, VAI unaccented condition: 0.988). Furthermore, the tonal height of the intonational peak pattern in accented words was 1.5 st below the group mean of 7 st. However, the analysis for the modulation of intensity revealed that PwPD13 did not differ from the group mean for this parameter (unaccented: $70 \mathrm{~dB}$, accented: $73 \mathrm{~dB}$ ). If one assumed that this patient is a good representative of a speaker with more advanced PD, one could hypothesize that as the disease progresses and the cognitive functions decline, the observed overmodulation of F0 and intensity switches to undermodulation, i.e. monopitch and monoloudness, which are frequently reported in the literature for patients with advanced PD (monoloudness: Countryman \& Ramig, 1993;Holmes et al., 2000; Ramig et al., 2001; monopitch: Darley et al., 1975; Ziegler \& Vogel, 2010).

Clinical Implications: The literature has already established clear links between vowel production and intelligibility in patients with only mild dysarthric symptoms

(Connaghan \& Patel, 2017; Kim et al., 2011; Skodda et al., 2011). Our research adds to the growing body of literature showing that a reduced vowel space as well as an overlap of vowel categories can already be considered as one part of the symptom complex of a beginning Parkinson's disease. Therefore, affected (back) vowel impairment acts as an early indicator for potential future speech impairments. Tongue function appears to be particularly prone to these effects, e.g. Hartelius \& Lillvik (2003) also reported that tongue function was more impaired than lip movements in a group of speakers with Multiple Sclerosis, with and without dysarthria. These findings further support the provision of therapeutic input at the early stages of speech impairment progression, whilst difficulties remain perceptually unnoticed, in order to preserve communication function better in the long term. They also highlight the need for more longitudinal studies that map the progression of speech symptoms over the course of the disease in the same detail as is currently available for other motor symptoms.

Second, our study is the first to report a relationship between speech production and the cognitive state. Whilst we were unable to conclude on the basis of our data why F0 and intensity modulation should be affected specifically by executive dysfunction (taskswitching); the findings open a new area of research both into underlying processes as well as clinical management. Our study suggests that the observed disturbances and their link to executive dysfunction could have been due to problems with resource allocation, which is a well-recognized deficit in PwPD. More research is necessary to identify how motor speech execution can be impacted by cognitive deficits, given their prevalence in PwPD. From a treatment point of view, a number of studies have found beneficial effects of cognitive training on gross motor symptoms such as gait in PwPD (Plotnik et al., 2005; Amboni et al., 2008). A study from Walton et al. (2018) investigated that gait disorders in PD are associated with cognition and that non-motor (cognitive) training can improve gait performance. Parallel investigations should be performed to assess whether speech production, as another axial symptom of PD, might also be improved by similar processes. The therapeutic consequence could be that cognitive training results in an improvement of (speech) motor symptoms in PwPD (cf. Salazar et al. 2017).

Limitations of the study: As is the case with most of the literature investigating speech in PwPD, we tested our participants only in the medication-ON condition. Consequently, our study design does not allow us to identify the influence of levodopa on speech 
production, and what are described as subclinical symptoms in this study could in fact manifest themselves with noticeable perceptual consequences in medication-OFF condition. However, the data still allow us to gather a representative picture of the influences of motor and cognitive functions on speech performance, given that all tasks were measured under the same condition. Furthermore, most of the PwPD included in our study showed signs of mild dysarthria, with only a few presenting with a rather moderate severity. However, this turned into an advantage in our study, as it allowed us to highlight disturbances at the phonetic level, which were not yet perceptually salient and could thus represent precursors to clinical impairment without being influenced by other factors such as rigidity. Future studies are necessary to understand how the speech system behaves in prosodic marking with a more progressed disease. Research on prominence marking in PwPD is helpful, because it involves coordination of several parameters rather than just looking at specific lip or tongue movement. Moreover, the experimental set-up should be carefully considered, so that the task is not too easy, for example by varying the prominence patterns or introducing a dual task element. The low variation between the stimuli could have influenced our results as the participants became used to the schemata. However, our research was still suited to highlight regulation and coordination issues, which are more likely to be impacted by cognitive decline.

\section{Conclusion}

This paper adds to the sparse literature on prominence marking in PwPD. It demonstrates that our cohort of PwPD could produce prosodic prominence by increasing pitch, syllable duration, intensity and adjusting their vowel production in terms of formant changes. Due to the mild impairment levels in our PwPD, not many of the speech variables showed significant group differences to the control participants, however, we identified a reduction in vowel space, leading to a decrease in acoustic vowel contrast, in line with measures of gross motor impairment (UPDRS III). In addition, the relationship between the four phonetic parameters was altered during prominence marking. Whilst syllable duration remained unaffected, the PwPD overused F0 and intensity in prominent positions, reflecting abnormalities in the regulatory mechanism for expressing prosodic prominence. Most interestingly, this behavior correlated with their level of executive functioning (task-switching). In contrast, working memory, attention and speed of information processing had no influence on the modulation of prosodic prominence. This is the first time that a link could be made between cognitive skills and speech production at the phonetic rather than language planning level.

\section{Acknowledgements}

We would like to thank all our participants for given their valuable time to this study. In addition, thanks go to Mark Ellison, Bodo Winter and Bastian Auris for statistical advice and data implementation.

\section{Funding}

This work was supported by the German Research Foundation (DFG) as part of the SFB 1252 "Prominence in Language" in the project A04 "Dynamic Modelling of Prosodic 
Prominence" at the University of Cologne, and a visiting professor stipend to Prof Lowit from the German Academic Exchange Service ("Deutscher Akademischer Austauschdienst (DAAD)"). 


\section{References}

Ackermann, H., \& Ziegler, W. (1991). Articulatory deficits in parkinsonian dysarthria: an acoustic analysis. Journal of Neurology, Neurosurgery \& Psychiatry, 54(12), $1093-$ 1098.

Ackermann, H., Hertrich, I., \& Hehr, T. (1995). Oral diadochokinesis in neurological dysarthrias. Folia Phoniatrica et Logopaedica, 47(1), 15-23.

Altmann, L. J., \& Troche, M. S. (2011). High-level language production in Parkinson's disease: a review. Parkinson's disease, 2011. DOI: http://dx.doi.org/10.4061/2011/238956

Amboni, M., Cozzolino, A., Longo, K., Picillo, M., \& Barone, P. (2008). Freezing of gait and executive functions in patients with Parkinson's disease. Movement disorders: official journal of the Movement Disorder Society, 23(3), 395-400.

Arbeitsgruppe Geriatrisches Assessment, 1997, Geriatrisches Basisassessment. Schriftenreihe Geriatrie Praxis, München: MMV Medizin Verlag.

Baese-Berk, M. \& Goldrick, M. (2009). Mechanisms of interaction in speech production. Language and Cognitive Processes, 24(4). 527-554. PMID: 19946622.

Bates, D., Maechler, M., Bolker, B. \& Walker, S. (2015). Fitting Linear Mixed-Effects Models Using lme4. Journal of Statistical Software, 67(1), 1-48.

Baumann, S. \& Röhr, C.T. (2015). The perceptual prominence of pitch accent types in german. In: The Scottish Consortium for ICPhS 2015 (ed.), Proceedings of the 18th International Congress of Phonetic Sciences, 298, 1-5. Glasgow, UK: The University of Glasgow.

Baumann, S. \& Winter, B. (2018). What makes a word prominent? Predicting untrained German listeners' perceptual judgments. Journal of Phonetics, 70, 20-38.

Baumann, S. (2014). The Importance of Tonal Cues for Untrained Listeners in Judging Prominence. Proc.10th ISSPCologne, 21-24.

Baumann, S., Becker, J., Grice, M., \& Mücke, D. (2007, August). Tonal and articulatory marking of focus in German. In: Proceedings of the 16th International Congress of Phonetic Sciences, Pirrot GmbH Dudweiler, 1029-1032.

Baumann, S., Grice, M., \& Steindamm, S. (2006, May). Prosodic marking of focus domainscategorical or gradient. In: Proceedings of speech prosody, 301-304.

Boersma, P., \& Weenink, D. (2014). Praat: sound pressure calibration. Retrieved 5 May from:

http://www.fon.hum.uva.nl/praat/manual/sound pressure calibration.html

Boersma, P., \& Weenink, D. (2018). Praat: doing phonetics by computer. [Computer program.] Version 6.0.37, retrieved 3 February 2018 from http://www.praat.org/. 
Braun, B., \& Ladd, D. R. (2003). Prosodic correlates of contrastive and non-contrastive themes in German. In: Speech Communication and Technology, 789-792.

Chafe, W. (1994). Discourse, Consciousness, and Time. Chicago: University of Chicago Press.

Cheang, H. S., \& Pell, M. D. (2007). An acoustic investigation of Parkinsonian speech in linguistic and emotional contexts. Journal of Neurolinguistics, 20(3), 221-241.

Cho, T. (2006). Manifestation of prosodic structure in articulatory variation: Evidence from lip kinematics in English. Laboratory phonology, 8, 519-548.

Clark, H. H. \& Haviland, S. E. (1977). Comprehension and the Given-New Contract. In: Roy Freedle (ed.), Discourse Production and Comprehension, 1-40. New Jersey: Ablex.

Cole, J. (2015). Prosody in context: a review. Language, Cognition and Neuroscience, 30(12), 1-31.

Connaghan, K. P. \& Patel, R. (2017). The Impact of Contrastive Stress on Vowel Acoustics and Intelligibility in Dysarthria. Journal of Speech, Language, and Hearing Research, 60(1), 38-50.

Countryman, S., \& Ramig, L. O. (1993). Effects of intensive voice therapy on voice deficits associated with bilateral thalamotomy in Parkinson disease: A case study. Journal of Medical Speech-Language Pathology, 1(4), 233-250.

D'Imperio, M. (2000). The role of perception in defining tonal targets and their alignment. Ph.D. thesis, Department of Linguistics, The Ohio State University.

Darley, F. L., Aronson, A. E., \& Brown, J. R. (1969). Differential diagnostic patterns of dysarthria. Journal of Speech, Language, and Hearing Research, 12(2), 246-269.

Darley, F. L., Aronson, A. E., \& Brown, J. R. (1975). Motor speech disorders. Saunders.

de Jong, K. (1995). The supraglottal articulation of prominence in English: Linguistic stress as localized hyperarticulation. The Journal of the Acoustical Society of America 97(1). 491-504.

de la Fuente-Fernández, R. (2012). Frontostriatal cognitive staging in Parkinson's disease. Parkinson's Disease, 2012. DOI: http://dx.doi.org/10.1155/2012/561046

De Looze, C., Moreau, N., Renié, L., Kelly, F., Ghio, A., Rico, A., ... \& Petrone, C. (2017). Effects of cognitive impairment on prosodic parameters of speech production planning in multiple sclerosis. Journal of neuropsychology. 1-24.

Dobinson, C. (2007). An investigation into the use of computers for conducting home practice of speech exercises for people with dysarthria. Unpublished PhD Thesis. Newcastle University, Newcastle upon Tyne.

Dromey, C. \& Benson, A. (2003). Effects of Concurrent Motor, Linguistic, or Cognitive Tasks on Speech Motor Performance. Journal of Speech, Language and Hearing Research, 46(5), 1234-1246.

Duffy, J. R. (2013). Motor Speech Disorders - Substrates, Differential Diagnosis, and Management. St. Louis, Elsevier, Mosby. 
Emre M, Aarsland D, Brown R, Burn DJ, Duyckaerts C, Mizuno Y, Broe GA, Cummings J, Dickson DW, Gauthier S, Goldman J, Goetz C, Korczyn A, Lees A, Levy R, Litvan I, McKeith I, Olanow W, Poewe W, Quinn N, Sampaio C, Tolosa E, Dubois, B. (2007). Clinical diagnostic criteria for dementia associated with Parkinson's disease B, Movement Disorders, 22(12), 1689-707. DOI: 10.1002/mds.21507

Fahn, S., Elton, R., \& Fahn, S. (1987). Members of the UPDRS development committee. Recent developments in Parkinson's disease, 2, Florham Park, 293-304.

Ferreira, F., \& Swets, B. (2002). How incremental is language production? Evidence from the production of utterances requiring the computation of arithmetic sums. Journal of Memory and Language, 46(1), 57-84. DOI: https://doi.org/10.1006/jmla.2001.2797

Filipe, M. G., Frota, S., \& Vicente, S. G. (2018). Executive functions and prosodic abilities in children with high-functioning autism. Frontiers in psychology, 9, 359, pp 1-10. DOI: https://doi.org/10.3389/fpsyg.2018.00359

Forrest, K., Weismer, G., \& Turner, G. S. (1989). Kinematic, acoustic, and perceptual analyses of connected speech produced by parkinsonian and normal geriatric adults. J Acoust Soc Am, 85(6), 2608-2622. DOI: 10.1121/1.397755

Gafos, A.I., Roeser, J., Sotiropoulou, S., Hoole, P. \& Zeroual, C. (2019). Structure in mind, structure in vocal tract. Natural Language \& Linguistic Theory. DOI: https://doi.org/10.1007/s11049-019-09445-y

Gaviria, A. M. (2015). Acoustic Realization of Contrastive Stress in Individuals with Parkinson's Disease. Unpublished master thesis, Louisiana State University.

Goetz, C. G., Tilley, B. C., Shaftman, S. R., Stebbins, G. T., Fahn, S., Martinez-Martin, P., et al. (2008). Movement Disorder Society-sponsored revision of the Unified Parkinson's Disease Rating Scale (MDS-UPDRS): Scale presentation and clinimetric testing results. Movement disorders, 23(15), 2129-2170.

Goldman, J. G., Holden, S., Ouyang, B., Bernard, B., Goetz, C. G., \& Stebbins, G. T. (2015). Diagnosing PD-MCI by MDS task force criteria: How many and which neuropsychological tests?. Movement Disorders, 30(3), 402-406.

Gómez-Vilda, P., Mekyska, J., Ferrández, J. M., Palacios-Alonso, D., Gómez-Rodellar, A., Rodellar-Biarge, V., . . Rektorova, I. (2017). Parkinson Disease Detection from Speech Articulation Neuromechanics. Frontiers in neuroinformatics, 11, 56. DOI: https://doi.org/10.3389/fninf.2017.00056

Gussenhoven, C. (2004). The Phonology of Tone and Intonation. Cambridge: Cambridge University Press.

Harrington, Jonathan, Janet Fletcher \& Mary E. Beckman. (2000). Manner and place conflicts in the articulation of accent in Australian English. In: Michael B. Broe \& Janet B. Pierrehumbert (Hrsg.), Bd. 5 (Papers in Laboratory Phonology), Kap. 4, 40 55. Cambridge: Cambridge University Press. 
Hartelius, L. and M. Lillvik (2003). "Lip and tongue function differently affected in individuals with multiple sclerosis." Folia Phoniatrica et logopaedica, 55(1), 1-9.

Hely MA, Reid WG, Adena MA, Halliday GM, Morris JG. (2008). The Sydney multicenter study of Parkinson's disease: the inevitability of dementia at 20 years. Movement Disorders, 23(6), 837-844.

Hinkle, D. E., Wiersma, W., \& Jurs, S. G. (2002). Applied statistics for the behavioral sciences. Boston: Houghton Miffln.

Ho, A. K., Iansek, R., \& Bradshaw, J. L. (1999a). Regulation of Parkinsonian speech volume: The effect of interlocuter distance. Journal of Neurology, Neurosurgery \& Psychiatry, $67(2), 199-202$.

Ho, A. K., Iansek, R., Marigliani, C., Bradshaw, J. L., \& Gates, S. (1999b). Speech impairment in a large sample of patients with Parkinson's disease. Behavioural neurology, 11(3), 131-137.

Hoehn, M. M., \& Yahr, M. D. (1998). Parkinsonism: onset, progression, and mortality. Neurology, 50(2), 318-318.

Holmes, R., M Oates, J., J Phyland, D., \& J Hughes, A. (2000). Voice characteristics in the progression of Parkinson's disease. International Journal of Language \& Communication Disorders, 35(3), 407-418.

Hornykiewicz, O. (1998). Biochemical aspects of Parkinson's disease. Neurology, 51(2 Suppl 2), 2-9.

Hsu, S. C., Jiao, Y., McAuliffe, M. J., Berisha, V., Wu, R. M., \& Levy, E. S. (2017). Acoustic and perceptual speech characteristics of native Mandarin speakers with Parkinson's disease. The Journal of the Acoustical Society of America, 141(3), EL293-EL299.

Jacobson, B. H., Johnson, A., Grywalski, C., Silbergleit, A., Jacobson, G., Benninger, M. S., \& Newman, C. W. (1997). The voice handicap index (VHI): development and validation. American Journal of Speech-Language Pathology, 6(3), 66-70.

Kalbe, E., Calabrese, P., Kohn, N., Hilker, R., Riedel, O., Wittchen, H. U., Dodel, R., Otto, J., Ebersbach, G. \& Kessler, J. (2008). Screening for cognitive deficits in Parkinson's disease with the Parkinson neuropsychometric dementia assessment (PANDA) instrument. Parkinsonism \& related disorders, 14(2), 93-101. DOI: https://doi.org/10.1016/i.parkreldis.2007.06.008

Kalbe, E., Rehberg, S. P., Heber, I., Kronenbuerger, M., Schulz, J. B., Storch, A., et al. (2016). Subtypes of mild cognitive impairment in patients with Parkinson's disease: evidence from the LANDSCAPE study. J Neurol Neurosurg Psychiatry, 87, 10991105.

Kalia, L.V. \& Lang, A.E. (2015). Parkinson's disease. The Lancet, 386, 896 - 912.

Kang, G. A., Bronstein, J. M., Masterman, D. L., Redelings, M., Crum, J. A., \& Ritz, B. (2005). Clinical characteristics in early Parkinson's disease in a central California 
population-based study. Movement disorders: official journal of the Movement Disorder Society, 20(9), 1133-1142.

Karlsson, F., \& Hartelius, L. (2019). How Well Does Diadochokinetic Task Performance Predict Articulatory Imprecision? Differentiating Individuals with Parkinson's Disease from Control Subjects. Folia Phoniatrica et Logopaedica, 1-10.

Kent, R. D., \& Kim, Y. J. (2003). Toward an acoustic typology of motor speech disorders. Clinical linguistics \& phonetics, 17(6), 427-445.

Kim, H., Hasegawa-Johnson, M., \& Perlman, A. (2011). Vowel Contrast and Speech Intelligibility in Dysarthria. Folia Phoniatrica et logopaedica, 63(4), 187-194.

Kochanski, G., Grabe, E., Coleman, J., \& Rosner, B. (2005). Loudness predicts prominence: Fundamental frequency lends little. The Journal of the Acoustical Society of America, 118(2), 1038-1054.

Koeslin, Jürgen. (2011). Psychiatrie und Psychotherapie für Heilpraktiker. München: Elsevier.

Kügler, F. (2008, May). The role of duration as a phonetic correlate of focus. In Proceedings of the Speech Prosody 2008 Conference, Editora RG/CNPq Campinas, Brazil, 591594.

Lawson, R.A., Yarnall, A.J., Duncan, G.W., Breen, D.P., Khoo, T.K., Williams-Gray, C.H., Barker, R.A., Burn, D.J. (2017). Stability of mild cognitive impairment in newly diagnosed Parkinson's disease. J Neurol Neurosurg Psychiatry, 88, 648-652.

Levelt, J. M. (1989). Speaking. From intention to articulation. Cambridge, MA: The MIT Press.

Levelt, W. J. M., \& Meyer, A. (2000). Word for word: Multiple lexical access in speech production. European Journal of Cognitive Psychology, 12, 433-452. DOI: https://doi.org/10.1080/095414400750050178

Lindblom, Björn. (1990). Explaining Phonetic Variation: A Sketch of the H\&H Thory. In William J. Hardcastle \& Alain Marchal (Hrsg.), Speech Production and Speech Modelling, 403-439. Dordrecht: Springer Netherlands.

Lowit, A., Brendel, B., Dobinson, C., \& Howell, P. (2006). An investigation into the influences of age, pathology and cognition on speech production. Journal of medical speech-language pathology, 14(4), 253-262.

Lowit, A., Ijitona, T., Kuschmann, A., Corson, S., \& Soraghan, J. (2018). What does it take to stress a word? Digital manipulation of stress markers in ataxic dysarthria. International journal of language \& communication disorders, 53(4), 875-887.

Maetzler, W., Liepelt, I., \& Berg, D. (2009). Progression of Parkinson's disease in the clinical phase: potential markers. The Lancet Neurology, 8(12), 1158-1171.

Mattingly, Ignatius G. (1981). Phonetic representation and speech synthesis by rule. In Terry Myers, John Laver \& John Anderson (Hrsg.), Advances in Psychology, 7, 415- 
420. Amsterdam: Elsevier Science. DOI: https://doi.org/10.1016/S0166$\underline{4115(08) 60217-4}$

Mücke, D., \& Grice, M. (2014). The effect of focus marking on supralaryngeal articulationIs it mediated by accentuation?. Journal of Phonetics, 44, 47-61.

Mücke, D., \& Grice, M. (2016). Segment und Geste in der Lautsprache. In Beatrice Primus \& Ulrike Domahs (eds.), Handbuch Laut, Gebärde, Buchstabe, Kap. 1, Berlin, Boston: De Gruyter Mouton, 3-24.

Mücke, Doris, Anne Hermes \& Taehong Cho. (2017). Mechanisms of regulation in speech: Linguistic structure and physical control system. Journal of Phonetics 64(Supplement C). 1-7. Mechanisms of regulation in speech.

Muir, R. T., Lam, B., Honjo, K., Harry, R. D., McNeely, A. A., Gao, F. Q., ... \& Zhou, X. J. (2015). Trail making test elucidates neural substrates of specific poststroke executive dysfunctions. Stroke, 46(10), 2755-2761.

Nelson, Noah Richard \& Wedel, Andrew. (2017). Th phonetic specifiity of competition: Contrastive hyperarticulation of voice onset time in conversational English. Journal of Phonetics 64(Supplement C). 51-70. Mechanisms of regulation in speech.

Penner, H., Miller, N., Hertrich, I., Ackermann, H., Schumm, F. (2001). Dysprosody in Parkinson's disease: an investigation of intonation patterns. Clinical Linguistics \& Phonetics, 15(7), 551-566.

Plotnik, M., Giladi, N., Balash, Y., Peretz, C., \& Hausdorff, J. M. (2005). Is freezing of gait in Parkinson's disease related to asymmetric motor function?. Annals of Neurology: Official Journal of the American Neurological Association and the Child Neurology Society, 57(5), 656-663.

Prieto, P. \& Torreira, F. (2007). The segmental anchoring hypothesis revisited: syllable structure and speech rate effects on peak alignment in Spanish. Journal of Phonetics, 35. 473-500.

Ramig, L. O., Sapir, S., Fox, C., \& Countryman, S. (2001). Changes in vocal loudness following intensive voice treatment (LSVT®) in individuals with Parkinson's disease: A comparison with untreated patients and normal age-matched control participants. Movement Disorders, 16(1), 79-83.

Rodgers, J. D., Tjaden, K., Feenaughty, L., Weinstock-Guttman, B., \& Benedict, R. H. (2013). Influence of cognitive function on speech and articulation rate in multiple sclerosis. Journal of the International Neuropsychological Society, 19(02), 173-180. DOI: https://doi.org/10.1017/S1355617712001166

Roy, N., Nissen, S. L., Dromey, C., \& Sapir, S. (2009). Articulatory changes in muscle tension dysphonia: evidence of vowel space expansion following manual circumlaryngeal therapy. Journal of communication disorders, 42(2), 124-135.

RStudio Team. (2016). RStudio: Integrated Development for R. RStudio, Inc., Boston, MA URL http://www.rstudio.com/ 
Rusz, J., Čmejla, R., Růžičková, H., Klempíř, J., Majerová, V., Picmausová, J., Roth, J. \& Růžička, E. (2013a). Evaluation of speech impairment in early stages of Parkinson's disease: a prospective study with the role of pharmacotherapy. Journal of Neural Transmission, 120(2), 319-329.

Rusz, J., Cmejla, R., Tykalova, T., Ruzickova, H., Klempir, J., Majerova, V., Picmausova, J., Roth, J.\& Ruzicka, E. (2013b). Imprecise vowel articulation as a potential early marker of Parkinson's disease: Effect of speaking task. The Journal of the Acoustical Society of America, 134(3), 2171-2181.

Salazar, R. D., Ren, X., Ellis, T. D., Toraif, N., Barthelemy, O. J., Neargarder, S., \& CroninGolomb, A. (2017). Dual tasking in Parkinson's disease: Cognitive consequences while walking. Neuropsychology, 31(6), 613.

Santangelo, G., Vitale, C., Picillo, M., Moccia, M., Cuoco, S., Longo, K., Pezzella, D., di Grazia, A., Erro, R., Pellecchia, M.T. (2015). Mild cognitive impairment in newly diagnosed Parkinson's disease: Alongitudinal prospective study. Parkinsonism Relat Disord, $21,1219-1226$.

Sapir, S., Ramig, L. O., Spielman, J. L., \& Fox, C. (2010). Formant centralization ratio: A proposal for a new acoustic measure of dysarthric speech. Journal of speech, language, and hearing research, 53(1), 114-125.

Sapir, S., Ramig, L., Spielman, J. \& Fox, C. (2011). Acoustic metrics of vowel articulation in Parkinson's disease: Vowel space area (VSA) vs. vowel articulation index (VAI). In Models and analysis of vocal emissions for biomedical applications. Firenze University Press. pp. 173-175.

Sapir, S., Spielman, J., Ramig, L. O., Hinds, S. L., Countryman, S., Fox, C., \& Story, B. (2003). Effects of intensive voice treatment (the Lee Silverman Voice Treatment [LSVT]) on ataxic dysarthria: A case study. American Journal of Speech-Language Pathology, 12(4), 387-399.

Scarborough, Rebecca. (2013). Neighborhood-conditioned pattrns in phonetic detail: Relating coarticulation and hyperarticulation. Journal of Phonetics 41(6). 491508.

Schretlen, D., Bobholz, J. H., \& Brandt, J. (1996). Development and psychometric properties of the Brief Test of Attention. The Clinical Neuropsychologist, 10(1), 80-89.

Sheikh, J. I., \& Yesavage, J. A. (1986). Geriatric Depression Scale (GDS): Recent evidence and development of a shorter version. Clinical Gerontologist: The Journal of Aging and Mental Health, 5(1-2), 165-173.

Skodda, S. (2015). Die Dysarthrie des Morbus Parkinson: Klinische Präsentation, pathophysiologische und diagnostische Aspekte. Sprache Stimme - Gehör, 39(4), 182-186. 
Skodda, S., Grönheit, W., \& Schlegel, U. (2012). Impairment of vowel articulation as a possible marker of disease progression in Parkinson's disease. PloS one, 7(2), e32132.

Skodda, S., Visser, W., \& Schlegel, U. (2011). "Vowel Articulation in Parkinson's disease." Journal of Voice, 25(4), e199-e205.

Stevens, K. N. (2000). Acoustic phonetics (Vol. 30). MIT press.

Swets, B., Desmet, T., Hambrick, D. Z., \& Ferreira, F. (2007). The role of working memory in syntactic ambiguity resolution: A psychometric approach. Journal of Experimental Psychology: General, 136, 64-81. DOI: https://doi.org/10.1037/0096-3445.136.1.64

Swets, B., Jacovina, M. E., \& Gerrig, R. J. (2013). Effects of conversational pressures on speech planning. Discourse Processes, 50(1), 23-51. DOI: https://doi.org/10.1080/0163853X.2012.727719

Tombaugh T.N. (2004). Trail Making Test A and B: Normative data stratified by age and education. Archives of Clinical Neuropsychology, 19, 203-214.

Tomlinson, C. L., Stowe, R., Patel, S., Rick, C., Gray, R., \& Clarke, C. E. (2010). Systematic review of levodopa dose equivalency reporting in Parkinson's disease. Movement disorders, 25(15), 2649-2653.

Tykalova, T., Rusz, J., Cmejla, R., Ruzickova, H., \& Ruzicka, E. (2014). Acoustic investigation of stress patterns in Parkinson's disease. Journal of Voice, 28(1), 129-136.

Tykalova, T., Rusz, J., Klempir, J., Cmejla, R., \& Ruzicka, E. (2017). Distinct patterns of imprecise consonant articulation among Parkinson's disease, progressive supranuclear palsy and multiple system atrophy. Brain and language, 165, 1-9.

van Heuven, V. J. J. P., \& Sluijter, A. M. (1996). Notes on the phonetics of word prosody. In: Goedemans, R., van der Hulst, H. \& Ellis Visch (Hrsg.) Stress Patterns of the World: Background. Holland Academic Graphics, The Hague, 233-269.

Vorperian, H. K., \& Kent, R. D. (2007). Vowel acoustic space development in children: A synthesis of acoustic and anatomic data. Journal of Speech, Language, and Hearing Research, 50(6), 1510-1545.

Walton, C. C., Mowszowski, L., Gilat, M., Hall, J. M., O’Callaghan, C., Muller, A. J., ... \& Naismith, S. L. (2018). Cognitive training for freezing of gait in Parkinson's disease: a randomized controlled trial. npj Parkinson's Disease, 4(1), 15.

Wechsler, D. (1997). Administration and scoring manual. San Antonio, TX: The Psychological Corporation.

Whitfield, J. A., \& Goberman, A. M. (2014). Articulatory-acoustic vowel space: Application to clear speech in individuals with Parkinson's disease. Journal of communication disorders, 51, 19-28. 
Wong, M. N., Murdoch, B. E., \& Whelan, B.-M. (2011). Lingual kinematics in dysarthric and nondysarthric speakers with Parkinson's disease. Parkinson's disease, 2011. doi:10.4061/2011/352838

Ziegler, W., \& Vogel, M. (2010). Dysarthrie: Verstehen, untersuchen, behandeln. Georg Thieme Verlag. 